\title{
Structure and function of the bacterial and fungal gut flora of Neotropical butterflies
}

2

*Author for correspondence: aravenscraft@gmail.com; tel 314-791-0738

${ }^{1}$ Department of Biology, Stanford University, Stanford, CA 94305 USA
Alison Ravenscraft* ${ }^{1,2}$, Michelle Berry ${ }^{1}$, Tobin Hammer $^{3}$, Kabir Peay ${ }^{1}$, Carol Boggs ${ }^{4}$

${ }^{2}$ Current address: Center for Insect Science, University of Arizona, Tucson, AZ 85721 US

${ }^{3}$ Ecology and Evolutionary Biology, University of Colorado Boulder, Boulder, CO 80309 USA

${ }^{4}$ Department of Biological Sciences, University of South Carolina, Columbia, SC 29208 USA

11

12 Author Contributions: AR, MB, KP and CB designed the experiments. AR and MB performed

13 the field work. AR conducted the lab work for the Illumina sequencing. AR, MB, and TH

14 analyzed the data. AR wrote the manuscript and all authors provided additional writing and

15 editorial feedback. 


\section{Abstract}

The relationship between animals and their gut flora is simultaneously one of the most common and most complex symbioses on Earth. Despite its ubiquity, our understanding of this invisible but often critical relationship is still in its infancy. We employed adult Neotropical butterflies as a study system to ask three questions: First, how does gut microbial community composition vary across host individuals, species and dietary guilds? Second, how do gut flora compare to food microbial communities? Finally, are gut flora functionally adapted to the chemical makeup of host foods? To answer these questions we captured nearly 300 Costa Rican butterflies representing over 50 species, six families and two feeding guilds: frugivores and nectivores. We characterized the bacteria and fungi in guts, wild fruits and wild nectars via amplicon sequencing and assessed the catabolic abilities of the gut flora via culture-based assays. Gut communities were distinct from food communities, suggesting that the gut environment acts as a strong filter on potential colonists. Nevertheless, gut flora varied widely among individuals and species. On average, a pair of butterflies shared $21 \%$ of their bacterial species and $6 \%$ of their fungi. Host species explained $25-30 \%$ of total variation in microbial communities while host diet explained 4\%. However, diet was still relevant at the individual microbe level — half of the most abundant microbial species differed in abundance between frugivores and nectivores. Diet was also related to the functional profile of gut flora: compared to frugivores, nectivores' gut flora exhibited increased catabolism of sugars and sugar alcohols and decreased catabolism of amino acids, carboxylic acids and dicarboxylic acids. Since fermented juice contains more amino acids and less sugar than nectar, it appears that host diet filters the gut flora by favoring microbes that digest compounds abundant in foods.

By quantifying the degree to which gut communities vary among host individuals, 
39 species and dietary guilds and evaluating how gut microbial composition and catabolic potential

40 are related to host diet, this study deepens our understanding of the structure and function of one

41 of the most complex and ubiquitous symbioses in the animal kingdom.

42

43 Key words: microbiota, Lepidoptera, diet, feeding guild, symbiosis, catabolism 


\section{Introduction}

45 Microbes have been detected in the gut of almost every animal studied to date. This ubiquity is underpinned by the myriad functions these microbes serve: Gut microbes can assist animals with the uptake, synthesis and recycling of nutrients, breakdown of toxic or recalcitrant chemicals, and resistance to pathogens (Dillon and Dillon 2004). Despite their prevalence and importance, our knowledge of how and why these symbiotic communities change across host individuals, species, functional ecological groups, and geographical locations is still in its infancy.

At the most basic level, variation in the community composition of the gut flora can result either from exposure of the host to different pools of potential microbial colonists

53 (including microbes transmitted vertically from parents to offspring), or from selective filtering of microbes by the physical and chemical conditions of the gut. Differences in diet or gut physiology between host dietary guilds, host species, and individual host organisms can affect both colonization by, and survival of, microbes in the gut.

Across disparate animal groups, host dietary guild has frequently attracted interest as a potential determinant of gut community composition. Different foods contain different microbial flora, and it is often assumed that consumption of foods can directly alter the composition of the gut community by introducing microbial colonists to the gut. This role appears to be most

61 important during initial colonization. Once established the gut community is normally resilient,

62 and most food-borne microbes pass through without becoming residents (e.g. Robinson et al

63 2010b; McNulty et al 2011). In addition to exposing hosts to alternative pools of microbes, diet

64 can alter the composition of both horizontally acquired and vertically transmitted gut microbes

65 by determining nutrient availability in, or affecting the chemical conditions of, the gut habitat

66 (Robinson et al 2010a). Ingestion of a particular nutrient will promote the growth of gut 
67 microbes that digest that nutrient, leading to feedbacks between diet and microbial community

68 function. For example, anaerobic fungi use lignocellulose as a food source; consumption of high

69 fiber foods by ruminant species leads to greater abundance of these fungi than in non-ruminants

70 (Gordon and Phillips 1998; Solomon et al 2016). These two mechanisms-food-borne microbial

71 colonists and the chemical composition of the diet — are generally expected to result in similar

72 gut flora among hosts that eat similar foods. Indeed, in many systems, gut community

73 composition has been shown to be more similar between species that eat similar diets, even when

74 these species belong to evolutionarily divergent groups (Colman et al 2012; Delsuc et al 2014).

75 For example, herbivorous, omnivorous, and carnivorous mammals and fish host gut communities

76 that are more similar within than between these three diet categories (Ley et al 2008a; Muegge et

77 al 2011; Sullam et al 2012). In insects, species belonging to the wood-feeding and detritivorous

78 guilds both host convergent gut flora (Colman et al 2012).

79 But host dietary guild is only one of many factors that may affect gut microbial

80 community composition. Differences in colonization can also result from living in different

81 habitats (e.g. Xiang et al 2006; Belda et al 2011), which results in sampling of divergent

82 microbial pools by hosts. Additionally, the chemical conditions of the gut vary among species,

83 independent of diet, and may act as a filter that selects for a specific gut community (Rawls et al

84 2006). For example, the guts of small animals, such as insects, range from fully aerobic to

85 anaerobic and therefore favor different sets of microbes (Johnson and Barbehenn 2000).

86 Similarly, gut $\mathrm{pH}$ varies among species and likely determines which microbes can establish in

87 the gut (Beasley et al 2015). The host immune system can also regulate potential gut colonists

88 (McFall-Ngai 2007; Salzman et al 2009). 
Gut flora vary among host species not only in the composition of the core community, but also in the degree of variation among individuals. Such intraspecies variation can result from

91 inter-individual differences in all of the factors mentioned above, including individual hosts'

92 diets, microbial source pools, gut chemistries, and immune systems. Species with the least

93 variable communities are often those that depend on their gut flora to survive on particularly

94 poor or recalcitrant diets, such as termites or herbivorous ants; these species also often have elaborately structured guts that both facilitate digestion and provide microenvironments that support a stable gut community (Engel and Moran 2013). In contrast, omnivorous hosts with simply-structured, tube-like guts, such as Drosophila, tend to have gut flora that vary more among individuals (Engel and Moran 2013). In general, gut membership is expected to be more

99 stable when the gut flora serve a more crucial function for their host.

101 gut microbial communities due to their variation in dietary guild, their abundance and species

102 diversity, and their often relatively simple gut flora. Further, insects are major primary

103 consumers, pollinators, and disease vectors in terrestrial ecosystems. Their gut flora may

104 therefore have a large, yet hidden, impact on ecosystem function (e.g. Nardi et al 2002). Indeed,

105 the ability of many insects to subsist on nutritionally unbalanced or recalcitrant foods often stems

106 directly from contributions of their gut flora (e.g. Warnecke et al 2007). Despite the importance

107 of, and increasing interest in, the insect gut flora in general, the adult lepidopteran microbiome

108 has been largely ignored. Although a few culture-based studies confirmed the presence of

109 bacteria in the adult gut decades ago (Steinhaus 1941; Kingsley 1972), culture-independent

110 profiling is only beginning. Such data exist for only a single species, Heliconius erato 
111 (Nymphalidae), and indicate that about 12 bacterial species dominate the adult gut (Hammer et al 112 2014).

113 In contrast to adults, the gut flora of several larval lepidopterans - particularly those of

114 economic importance — have been characterized (Broderick et al 2004; Robinson et al 2010b;

115 Pinto-Tomás and Sittenfeld 2011). However, the adult gut flora differ from those of larvae

116 (Hammer et al 2014). This difference may derive from the fact that during pupation, the contents

117 of the guts are voided, antimicrobial peptides are secreted into the gut lumen, and gut itself is

118 replaced, presumably eliminating a large portion of the larval gut flora (Russell and Dunn 1996;

119 Hakim et al 2010; Johnston and Rolff 2015). Indeed, in a culture-based study, Kingsley (1972)

120 found that the density of colony-forming units derived from the guts of monarch butterflies

121 (Danaus plexippus) decreased 1000-fold between pupae and freshly emerged adults. Existing

122 data on larval lepidopterans therefore do not shed light on the gut flora of adult butterflies.

123 Butterflies are biologically and economically important as herbivores and pollinators, and in

124 areas outside of gut community ecology they are well-studied model organisms in the fields of

125 population and nutritional ecology (Boggs et al 2003). Characterization of the causes and

126 consequences of variation in the adult lepidopteran gut flora will therefore pave the way for

127 connections between microbiome research and classical questions in ecology and evolutionary

128 biology.

129 Here we use adult butterflies as a novel study system to characterize patterns of variation

130 in the gut flora at the level of host individuals, species, and feeding guilds. We focus on how host

131 nutritional ecology affects the community composition and functional capacity of the gut flora.

132 The Neotropical community of butterflies allows for a high degree of replication both within and

133 between species while controlling for geographic origin. Neotropical butterflies also exhibit 
134 well-described ecological variation that likely structures the gut community, particularly in

135 feeding behavior: adults of some species feed on nectar, while others feed on the juice of rotting

136 fruits. Adults are easily captured in the wild, permitting examination of the natural gut flora and

137 avoiding the microbial community shifts frequently observed in captive animals (e.g. Chandler et

138 al 2011). These characteristics make Neotropical butterflies a useful system to elucidation

139 patterns in the composition and function of the gut microbial community.

141 How does gut microbial community composition vary among individuals, species, and dietary

142 guilds? (3) How does gut microbial composition compare to that of butterfly foods? (4) How

143 does community composition translate into functional abilities — specifically, catabolism of

144 various carbohydrates and amino acids?

146 expected butterfly gut communities to cluster according to both host diet and host species, such

147 that microbial beta diversity would be lowest between conspecific hosts, intermediate between

148 hosts that belong to different species but the same feeding guild, and highest between

149 heterospecific hosts that belong to different feeding guilds. We hypothesized that we would

150 observe signals of environmental acquisition of the gut flora. Specifically, we predicted that most

151 of the gut community would be a subset of the microbes present in the host's food, and that

152 frugivores, which feed on more "contaminated" foods (e.g. rotting fruit), would have more dense

153 gut communities than nectivores. Finally, we hypothesized that the digestive abilities of the gut

154 community would differ between feeding guilds, and that gut community catabolism should be

155 highest for nutrients abundant in the host's diet. 
157 Methods

158 Study site

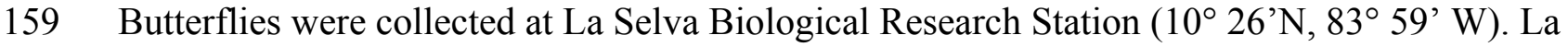

160 Selva is located in the lowlands on the Caribbean versant of the Cordillera Central in Costa Rica.

161 The station owns 1,600 hectares, $55 \%$ of which is primary tropical wet forest; the remainder

162 comprises secondary forest and disturbed habitats (e.g. abandoned pastures and plantations) in

163 various states of regrowth. This habitat diversity allowed us to collect a large diversity of

164 butterfly species while controlling for the geographic origin of our samples. La Selva also

165 provided the facilities necessary for both the culture-dependent and -independent components of

166 our project (e.g. a wet lab and laminar flow hood). From January to March 2013, we captured

167 butterflies and sampled butterfly foods along the trail system in primary forest, secondary forest

168 and recovering pastures. We returned to La Selva in March 2014 to collect additional food

169 samples (fruits and nectars).

\section{Study organisms}

172 Neotropical lepidopterans belong to two main feeding guilds: nectar feeders and fruit feeders.

173 The Papilionidae, Pieridae, Lycaenidae, Riodinidae, Hesperiidae, and some Nymphalidae feed

174 primarily on flower nectar, while several subfamilies of the Nymphalidae - Satyrinae,

175 Morphinae, Charaxinae, and some members of the Nymphalinae — feed primarily on rotting

176 fruits or other non-floral liquids (DeVries 1987, DeVries 1988). Our sampling included

177 representatives of all of the nectivorous families - though most individuals derived from the

178 Pieridae and Nymphalidae - as well as representatives from all the frugivorous nymphalid 
subfamilies described above (Supplementary Table 1). Three Heliconius (Nymphalidae:

Heliconiinae) species were sampled; this genus supplements its nectivorous diet with pollen.

\section{Sample collection and processing}

We used aerial nets to catch both nectivorous and frugivorous species. To capture frugivores we also used Van Someren-Rydon traps (Daily and Ehrlich 1995) baited with a fermented mixture of fruit (primarily mango), molasses and rum. Traps were checked at least once every two days. Upon capture, butterflies were placed in glassine envelopes and transported to the lab. We identified butterflies to species using The Butterflies of Costa Rica and Their Natural History (Devries 1987). Butterflies were fed a 5 to 10 microliter droplet of a filter-sterilized solution of sugar and nigrosin dye (Sigma 198285) to assist with visualization of their guts during dissection. Butterflies were euthanized with ethyl acetate, washed with $70 \%$ ethanol, and dissected in a laminar flow hood to minimize contamination with environmental bacteria and fungi. Guts were removed and homogenized in a small amount of inoculating fluid (described below) in a sterile $2 \mathrm{~mL}$ tube. A portion of the homogenate was cultured to assess gut community catabolic profile (described below). The remainder of the gut homogenate was suspended in cetyl trimethyl ammonium bromide $(\mathrm{CTAB})$ and refrigerated at $4^{\circ} \mathrm{C}$ until transportation to Stanford University for DNA extraction. CTAB is an effective preservative for storage of insect samples prior to microbiota analysis (Hammer et al 2015).

We also collected samples of the butterflies' foods to characterize potential source pools of microbial gut communities. Trap baits were sampled in 2013 and 2014 by suspending approximately 0.25 to $0.5 \mathrm{~mL}$ of rotten fruit slurry in CTAB. In 2014, nectar samples were also collected. We used capillary tubes to extract nectar from flowers in the field, obtaining between 
0.5 and $10 \mu \mathrm{L}$ per flower. Nectars were also preserved in CTAB. We only collected nectar from species at which we directly observed at least one instance of butterfly feeding. In 2013, we

204 sampled fruits of Dipteryx oleifera (Fabaceae) by scraping flesh from the surface of the fruit into

205 CTAB. In 2014, we rinsed the fruit with $5 \mathrm{~mL}$ of sterile water and preserved 0.25 to $0.5 \mathrm{~mL}$ of

206 the rinsate, since butterflies only feed on surface juices and do not consume the pulp. Dipteryx

207 oleifera is the second-most abundant leguminous tree in primary forest at La Selva (McDade et

208 al 1994) and was the only wild fruit we observed butterflies feeding upon during both visits to

209 the site.

210 Research was carried out under permit numbers 202-2012-SINAC and R-016-2014-OT-

\section{CONAGEBIO.}

\section{Community catabolic profiling}

214 A portion of each gut homogenate was seeded into $10 \mathrm{~mL}$ of inoculating fluid (IF-A, Biolog

215 Catalog No. 72401) and cultured in microbial catabolic phenotype plates (Gen III microplates,

216 Biolog Inc., Hayward, CA). The volume of gut homogenate used depended on the size of the

217 butterfly: we used 25 microliters for most animals, but took less volume from the largest species

218 (whose gut homogenate was more concentrated, since the guts themselves were large), and more

219 volume from the smallest species (whose gut homogenate was less concentrated, since the guts

220 were small). This was meant to correct for the grossest differences in inoculation density; further

221 corrections are described below.

222 The catabolic phenotyping plates allowed simultaneous assessment of catabolism of 71

223 carbon and nitrogen sources (e.g. glucose, fructose, ammonia, uric acid; Table S5) via a

224 colorimetric assay. Plates were incubated at ambient temperature. Absorbance at $590 \mathrm{~nm}$ was 
225

226

measured twice per day using a 96-well plate reader (Chromate-4300, Awareness Technology Inc., Palm City, FL) until color remained stable (5 days on average).

To reduce bias due to differences in inoculum density between the samples, the data were standardized as in Garland et al (2001). Briefly, based on visual inspection of the distribution of plate averages, we chose a target average absorbance value per plate of 190. For each plate, we selected data from the timepoint at which the average absorbance was closest to this target.

These measurements were standardized by subtracting the value of the negative control and dividing by the plate's average absorbance.

\section{Microbial community characterization}

Samples were shipped to Stanford University, where they were homogenized via bead beating, extracted with chloroform, and cleaned using the DNeasy Blood and Tissue Kit (Qiagen, Germantown, MD). Our DNA extraction protocol is described in detail in Peay et al (2007). Bacterial DNA was amplified with primer set 515f (5'-GTGCCAGCMGCCGCGGTAA3') and 806r (5'- GGACTACHVGGGTWTCTAAT-3'), which amplifies the V4 hypervariable region of the $16 \mathrm{~S}$ rRNA with few taxonomic biases (Bergmann et al 2011). The bacterial amplicons were indexed with barcoded forward and reverse primers to (Caporaso et al 2012;

Kozich et al 2013). The PCR reaction contained $0.2 \mu \mathrm{M}$ forward primer, $0.2 \mu \mathrm{M}$ reverse primer, 0.2 mM dNTP, 0.65 U OneTaq HotStart (New England Biolabs) and 1X Thermopol buffer (New England Biolabs) in a volume of $25 \mu \mathrm{L}$. The thermocycler program began with denaturation at $94 \mathrm{C}$ for 3 minutes followed by 35 cycles of denaturation at $94 \mathrm{C}$ for 45 seconds, annealing at 50 $\mathrm{C}$ for 60 seconds, and extension at $68 \mathrm{C}$ for 90 seconds, with a final extension of $68 \mathrm{C}$ for 10 minutes (adapted from the Earth Microbiome Project protocol; Gilbert et al 2014). 
Fungal DNA was amplified with primers ITS1f (5'-

CTTGGTCATTTAGAGGAAGTAA-3') and ITS2 (5'-GCTGCGTTCTTCATCGATGC-3')

250 (White et al 1990; Gardes and Bruns 1993). The reverse primer was barcoded to index the

251 samples (Smith and Peay 2014). The PCR recipe was identical to the bacterial PCR recipe above.

252 Thermocycler conditions were denaturation at $95 \mathrm{C}$ for 1 minute followed by 35 cycles of $94 \mathrm{C}$

253 for 30 seconds, $52 \mathrm{C}$ for 60 seconds, and $68 \mathrm{C}$ for 60 seconds, with a final extension of $68 \mathrm{C}$ for

2545 minutes. Each bacterial and fungal amplification was run in triplicate to minimize the effects of 255 stochastic amplification.

(Agencourt A63881) and the final concentration of each sample was quantified fluorometrically with a 96-well plate reader (Qubit dsDNA HS kit, Thermo Fisher Q32854). An equal mass of

DNA from each sample was added to the bacterial or fungal library, respectively. Bacterial and

260 fungal libraries were sequenced in separate runs on an Illumina MiSeq platform at the Stanford

261 Functional Genomics Facility (Stanford, CA), with 2 × 250 chemistry for bacteria and 2 x 300

262 chemistry for fungi.

\section{Illumina sequence data processing and cleaning}

265 We used the program cutadapt (Martin 2011) to remove priming sites and poor quality bases at

266 the 5' and 3' ends of the sequences. Sequences were merged and clustered at a 97\% similarity

267 cutoff with UPARSE (Edgar 2013). Both de-novo and reference-based chimera checking were

268 performed in UPARSE; bacterial reads were compared to the RDP Gold database and fungal

269 reads to the UNITE database. Bacterial taxonomy was initially assigned using the RDP classifier

270 (Wang et al 2007) with Greengenes (McDonald et al 2012) as the training set. We used the RDP 
271 classifier with the Warcup (Deshpande et al 2016) training set to assign fungal taxonomy. We

272 checked and revised these assignments by aligning our representative sets of bacterial and fungal

273 sequences against the NCBI nucleotide collection using BLAST.

274 OTUs that were identified as lepidopteran 18S, archaeans, mitochondria, or chloroplasts

275 were excluded. (These accounted for less than $0.1 \%$, less than $0.1 \%, 0.6 \%$, and $4.8 \%$ of the raw

276 total reads, respectively, and 98\% of the chloroplast reads derived from samples of butterflies'

277 foods.) Additionally, DNA extraction kits and other laboratory reagents are known to contain

278 microbial DNA that can contaminate microbiome analyses (Salter et al 2014). We found fourteen

279 bacterial OTUs that were present at higher abundance in the negative controls than the butterfly

280 gut samples (Supplementary Table 3); these were classified as contaminants and removed from

281 the dataset prior to statistical analyses. The fungal dataset (especially nectivore samples)

282 contained many ectomycorrhizal lineages that were unlikely to be members of the butterfly gut

283 community. This suggested that the butterfly gut samples (particularly those of nectivores) had

284 low fungal biomass, resulting in detection of ambient lab and environmental contaminants. We

285 removed lineages that were likely contaminants from the analysis: we omitted 135 OTUs

286 assigned to the genus Mortierella, which are decay fungi that inhabit soil. Wood decomposers

287 and ectomycorhizal, arbuscular or ericoid mycorrhizal fungi were also removed from the dataset,

288 as were lineages that were present at higher abundance in the negative controls than in the

289 samples (Supplementary Table 4). We retained lineages that were known phylloplane

290 inhabitants, plant pathogens, and fruit decomposers, as well as most ascomycete and

291 basidiomycete yeasts.

292 Frugivorous butterflies were captured either with traps baited with fermented fruit or with

293 aerial nets. For those captured in baited traps, a portion of the gut flora likely derived from 
microbes in the trap baits. To identify these microbes, we tested for differential abundance of bacterial and fungal OTUs between trapped frugivores and netted frugivores using the $m t$

296 function in the R package "phyloseq" (McMurdie and Holmes 2013), which includes an FDR 297 correction for multiple testing. No bacterial OTUs differed in abundance between netted and

298 trapped animals, but three fungal OTUs were significantly more abundant in the trapped

299 butterflies. These OTUs were all strains of the yeast Kazachstania exigua, which was also by far

300 the most abundant fungus detected in the traps. These three OTUs accounted for $43.7 \%$ of all

301 fungal reads from the fermented baits and only $0.3 \%$ of reads from wild fruits. We concluded

302 that the OTUs had been introduced into the trapped butterflies' guts via the fermented baits and

303 removed them from the data derived from butterfly samples.

304 To control for differences among samples in sequencing depth, we rarefied the

305 sequencing data using phyloseq. Bacteria were rarefied to 1000 and fungi to 200 sequences per

306 sample. These cutoffs allowed us to retain as many samples in the dataset as possible while still

307 profiling the dominant microbes. All sequencing results reported are based on rarefied data,

308 unless otherwise noted.

310 Quantification of total bacterial abundance

311 Bacterial DNA was quantified with qPCR using SYBR green fluorescent chemistry (iCycler IQ,

312 Bio-Rad, Hercules, CA). Since the 515f/806r primer set sometimes amplifies butterfly $18 \mathrm{~S}$ in

313 addition to bacterial $16 \mathrm{~S}$ rRNA, we designed a PNA clamp to block 18S amplification (Lundberg

314 et al 2013). The clamp sequence was GCCCGCTTTGAGCACTCT and it was synthesized by

315 PNA Bio (Thousand Oaks, CA). The reaction volume was $20 \mu \mathrm{L}$ and the recipe was $7.5 \mu \mathrm{M}$

316 PNA clamp, $0.2 \mu \mathrm{M}$ 515f primer, $0.2 \mu \mathrm{M}$ 806r primer, and 1X PerfeCTa SYBR Green FastMix 
317 for iQ (Quanta Biosciences, Gaithersburg, MD). Thermocycler settings were an initial 10-minute

318 denaturation at $95 \mathrm{C}$ followed by 45 cycles of denaturation at $95 \mathrm{C}$ for $15 \mathrm{sec}$, PNA clamp

319 annealing at $76 \mathrm{C}$ for $10 \mathrm{sec}$, primer annealing at $50 \mathrm{C}$ for $30 \mathrm{sec}$, and extension at $68 \mathrm{C}$ for 30

320 sec. To check that amplified fragments were the expected length, we performed a melt curve

321 ramping from $55 \mathrm{C}$ to $95 \mathrm{C}$ in $0.5 \mathrm{C}$ increments at 10 second intervals. PCR products were also

322 visualized using gel electrophoresis.

323 To calculate the starting number of 16S rRNA copies, each sample's threshold cycle was

324 compared to an internal standard curve ranging from an initial 10 to $10^{7}$ copies per $\mu \mathrm{L}$ of $E$. coli

325 16S rRNA amplicons. (Generation of these standards is described below.) Each sample and

326 standard was run in triplicate and the results were averaged. The correlation coefficients of the

327 standard curves for all qPCR plates ranged from 0.973 to 0.988 .

328 Since a fraction of each sample was removed for culture-based catabolic profiling prior to

329 DNA preservation and extraction, the qPCR estimates were corrected accordingly. For example,

330 if $50 \%$ of the gut homogenate from a given sample was removed prior to DNA preservation, that

331 sample's qPCR estimate was multiplied by two to obtain the estimated total 16S rRNA count for

332 the entire gut.

The 16S rRNA standard curve was generated as follows: 16S rRNA was amplified from

334 E. coli using primers $27 \mathrm{f}$ and 1492 r, ligated into a plasmid vector, and cloned into chemically

335 competent cells. Colonies were screened for inserts of the expected size by PCR amplification

336 using M13f and M13r primers followed by gel electrophoresis. To further screen for the correct

337 insert, several colonies were bidirectionally sequenced. One colony that passed both screens was

338 selected for the standard curve. This clone was grown to saturation in LB + kanamycin selective

339 media. Plasmids were extracted using the Qiagen Plasmid Mini kit and linearized using the 
340 restriction enzyme Spel (FastDigest, Thermo Fisher FD1253). DNA was purified using a Qiagen

341 PCR cleanup column and run on a gel to verify complete linearization. DNA concentration was

342 quantified with PicoGreen and copy number per $\mu \mathrm{L}$ was calculated as the molecular weight of

343 plasmid plus insert (g/molecule, calculated as length in bp * $660 \mathrm{Da} / \mathrm{bp} / 6.02 * 10^{23}$ ) divided by

344 the measured DNA concentration $(\mathrm{g} / \mu \mathrm{L})$. Via serial dilution of the raw extract with sterile TE,

345 we generated a standard curve ranging from $10^{7}$ copies $/ \mu \mathrm{L}$ to 10 copies $/ \mu \mathrm{L}$.

346 We were not able to quantify total fungal abundance because our ITS primers amplified

347 fragments of vastly different lengths (ranging from 250 base pairs to over $1000 \mathrm{bp}$ ).

\section{Statistical analyses}

350 All models had Gaussian error structure and were fit in R version 3.2.2 (R Core Team 2015).

351 Models without random effects were fit using the $l m$ command in the "stats" package. Models

352 with random effects were fit using the lmer command in the package "Ime4" (Bates et al 2014).

\section{a. Variation in total bacterial load among host species and feeding guilds}

355 A $\log$ transformation was applied to the $16 \mathrm{~S}$ rRNA counts prior to analysis. We used linear and

356 linear mixed effect models (Zuur et al 2009) to assess whether total bacterial load differed

357 among host species or between host feeding guilds. We used a likelihood ratio test to compare

358 models with and without a random effect of host species, including fixed effects of host diet,

359 wing length (as a proxy for the animal's size), and capture date. We then tested for significance

360 of the fixed effects using backwards model selection with likelihood ratio tests, including a

361 random effect of host species in all models. 


\section{b. Variation in OTU richness}

364 To test whether observed bacterial and fungal richness differed among host species, we again

365 used a likelihood ratio test to compare models with and without a random effect of host species,

366 including a fixed effect of host diet to control for overall diet-based differences. We tested for

367 correlation between host diet and observed bacterial and fungal richness by comparing models

368 with a fixed effect of diet to models without the fixed effect; both models had a random effect of

369 host species. We omitted data from host species represented by a single individual from these 370 analyses.

\section{c. Variation in microbial community composition}

374 microbial community composition, we used ordination (NMDS) plots to visualize and

375 perMANOVAs to test for dissimilarity in microbial community composition (Anderson 2001).

376 Both operations were performed on the Bray-Curtis dissimilarities between rarefied samples. We

377 performed two perMANOVAs (the adonis test in the R package "vegan"; Oksanen et al 2015) to

378 test for dissimilarity in bacterial and fungal communities, respectively. These tests included

379 terms for both host diet and host species, thus simultaneously testing whether gut communities

380 differed between host species and feeding guilds. Host species represented by a single individual

381 were omitted from these analyses.

382 If communities differ in variance, perMANOVA results can be unreliable (Anderson and

383 Walsh 2013). To verify the results of the tests, we therefore tested for differences in dispersion

384 among feeding guilds using the betadisper function from the package "vegan." For bacteria, the

385 feeding guilds did not differ in dispersion (anova: $d f=1, \mathrm{~F}=0.12, \mathrm{p}=0.73$ ) therefore the data 
conformed to the assumptions of perMANOVA. For fungi, the feeding guilds did differ in spread (anova: $d f=1, \mathrm{~F}=4.4, \mathrm{p}=0.04$ ). However, after removing the five host species with extreme dispersions, the feeding guilds no longer differed in spread (anova: $d f=1, \mathrm{~F}=1.3, \mathrm{p}=0.21$ ). The perMANOVA results with these five species removed were qualitatively identical and numerically similar to those obtained from all host species, therefore we report the test results for all species (omitting those represented by a single individual).

In order to understand the effect of host diet on individual OTU abundances, we modeled the relative abundance of a bacterial or fungal OTU (counts out of 1000 or 200, respectively) as a function of the interaction between host diet and OTU identity, with a random effect of host species. Since it was not feasible to model abundances of every OTU, we selected the 20 bacterial or 20 fungal OTUs that were present at the highest abundances in the pooled data and were detected in at least $10 \%$ of either frugivore or nectivore samples. Prior to the analyses, abundance data were transformed by $\log (\mathrm{x}+1)$ to homogenize variance.

Among host species: We used perMANOVAs (described above) to test for dissimilarity in the gut flora among host species while accounting for differences between feeding guilds. Since perMANOVA assumes homogeneity of variance among communities, we again used the betadisper function to test for differences in dispersion. Host species' bacterial and fungal communities did differ in dispersion (bacterial anova: $d f=36, \mathrm{~F}=2.4, \mathrm{p}<0.001$; fungal anova: $d f$ $=28, \mathrm{~F}=11.4, \mathrm{p}<0.001)$. For bacteria the difference was driven by five species that had unusually low variance; when these were removed, the remaining 32 species no longer differed in spread (anova: $d f=31, \mathrm{~F}=0.9, \mathrm{p}=0.65$ ). For fungi, the difference was also driven by five extreme species. The remaining 24 species in the fungal dataset no longer differed in variance (anova: $d f$ $=23, \mathrm{~F}=1.3, \mathrm{p}=0.16$ ). For both bacteria and fungi, we re-ran the perMANOVA tests using the 
subset host species that did not differ in dispersion. Results were again qualitatively the same as those for all host species, so we report the results for all species. calculated the median percentage of OTUs shared between individuals, where the percentage

413 shared between individuals A and B was calculated as $0.5 *$ (number of A's OTUs shared by B / 414 total OTUs in A + number of B's OTUs shared by A / total OTUs in B). Between food microbial communities and gut communities: We used NMDS plots (on

416 Bray-Curtis distances between rarefied samples) to visualize differences between the butterfly 417 gut flora and the microbial composition of butterfly foods. To test whether gut flora were more 418 similar to the microbial communities of one food than to another food, we calculated the 419 pairwise Bray-Curtis distances between each butterfly gut community and each food community and performed t-tests on the per-butterfly averages of these distances for the three foods (fruits, nectars and baits). To correct the resulting p-values for multiple testing, we used the BenjaminiHochberg false discovery rate (FDR) method as implemented by the p.adjust function in $\mathrm{R}$

423 (Benjamini and Hochberg 1995).

\section{d. Variation in microbial community function}

426 The Hellinger transformation was applied to the standardized catabolic data prior to all analyses.

428 function were correlated with differences in microbial species composition, Mantel tests were 429 performed between the Bray-Curtis distances between bacterial or fungal microbial communities 430 and the Euclidean distances between catabolic profiles. Since trap bait-derived fungal OTUs 431 (three strains of Kazachstania exigua) were introduced into the Biolog plates via frugivores' 
432 guts, these OTUs were included in the calculation of Bray-Curtis distances between fungal

433 communities. However, results were qualitatively equivalent when these three OTUs were

434 omitted from the Bray-Curtis calculations.

With host species and diet: First, we tested for correlation between gut community

436 catabolic profile and both host diet and host species by performing a perMANOVA on the

437 Euclidean distances between the gut catabolic profiles. (Singleton host species were omitted

438 from this analysis.) To investigate differences between frugivores and nectivores in more detail,

439 we next modeled substrate catabolism as a function of the interaction between host diet and

440 substrate identity, including a fixed effect for the log of the number of 16S rRNA copies in the

441 host's gut to control for differences in bacterial density, and a random effect for the identity of

442 the host butterfly to control for overall differences in catabolic activity of the gut community

443 among individuals. Finally, to test whether catabolism of certain classes of substrate (for

444 example, amino acids or sugars) was consistently up- or down-regulated with host diet, we

445 modeled substrate catabolism as a function of the interaction between host diet and substrate

446 class, with a fixed effect for $\log (16 \mathrm{~S}$ rRNA counts) and a random intercepts of host identity as

447 before, plus an additional random intercept and random diet effect (slope term) for each

448 substrate. Thirteen substrates belonged to classes with three or fewer substrates and were

449 therefore omitted from the class-level analysis. For both models, least-square means and the

450 contrasts between them were calculated using the lsm command in the "lsmeans" package (Lenth

451 2016) to assess the whether a substrate or substrate class was catabolized differently between

452 frugivores and nectivores. The resulting p-values for each substrate or substrate type,

453 respectively, were FDR corrected to control for multiple testing. 


\section{Results}

456 Below we first describe the overall composition of the adult butterfly gut flora, then address

457 patterns of variation in microbial community composition at the level of host feeding guild, host

458 species, and host individual. Gut communities are then compared to the microbes present in

459 butterfly foods. Functional differences between gut microbial communities are described last.

\section{Bacteria and fungi present in the butterfly gut}

We sequenced the bacterial flora of 306 butterflies, of which 290 were retained after

463 rarefaction, and the fungal gut communities of 247 butterflies, 161 of which were retained after

464 rarefaction. (See Table 1 for a full sample size breakdown and Supplementary Table 1 for counts

465 per butterfly host species). After sequence processing, quality filtering, and removal of

466 contaminant OTUs, 7.2 million bacterial sequences and 1.5 million fungal sequences were

467 obtained from butterfly guts. After rarefying, a total of 958 bacterial OTUs and 880 fungal OTUs

468 were observed across all butterflies. (Note that numbers of bacterial and fungal OTUs cannot be

469 compared because bacteria and fungi were rarefied to different read depths.) Individual

470 butterflies hosted a mean of 30 (interquartile range:18-37) bacterial OTUs (number observed at a

4711000 read per sample cutoff) and 21 (IQR: 12-28) fungal OTUs (number observed at a 200 read

472 per sample cutoff).

473 The most prevalent bacterial phyla and classes across all butterflies were

474 Proteobacteria:Gammaproteobacteria (40\% of all butterfly-derived bacterial sequences),

475 Proteobacteria:Alphaproteobacteria (28\%), Firmicutes:Bacilli (12\%),

476 Bacteroidetes:Flavobacteriia (7\%), and Tenericutes:Mollicutes (4\%). The dominant fungal class

477 was Saccharomycetes (62\% of all butterfly-derived fungal sequences) in the phylum 
Ascomycota. All of these Saccharomycetes belonged to the order Saccharomycetales, which

Basidiomycota:Tremellomycetes (8\%), Zygomycota:Mucoromycotina (6\%),

Ascomycota:Dothideomycetes (6\%) and Basidiomycota:Microbotryomycetes (5\%). which are known to associate with insects and/or plants — particularly nectars, decaying fruits, and beetle guts (Suh et al 2005; Kurtzman et al 2011). One OTU was most closely identified as a Rhizopus species; this genus is commonly associated with decaying vegetable matter (Kirk et al 2008). Likely plant pathogens (e.g. Clavariopsis sp. and a Letiomycetes species) and plantassociated basidiomycotous yeasts (OTUs that aligned most closely to the genera Bensingtonia) were also common. 
$50173 \%$. The most prevalent fungal OTUs were Hanseniaspora uvarum found in 45\%,

502 Hanseniaspora opuntiae found in 42\%, and Hanseniaspora guilliermondii found in 40\%.

503

\section{1a. Total bacterial load in the butterfly gut}

505 We quantified the total abundance of bacteria in the guts of 261 butterflies. The total number of

$50616 \mathrm{~S}$ rRNA copies in a butterfly's gut ranged from $5 \times 10^{5}$ to $1 \times 10^{11}$, with a median of $7.5 \times 10^{8}$

507 (interquartile range: $1.2 \times 10^{8}-2.7 \times 10^{9}$ ). Larger butterfly individuals hosted greater numbers of

508 bacteria (Supplementary Figure 1). Bacterial load differed among host species (Figure 1; random

509 host species intercept term: $\left.d f=1, \chi^{2}=17.5, p<0.001\right)$ but did not differ between feeding guilds

510 after accounting for host species and size (fixed diet term: $d f=1, \chi^{2}=1.8, p=0.18$ ).

511

512 2. Variation in gut microbial community composition between host dietary guilds, species, and

513 individuals.

514 2a. Variation between the feeding guilds

515 The gut flora of frugivores and nectivores did not differ from each other in observed OTU

516 richness (Figure 2; bacteria fixed feeding guild term: $d f=1, \chi^{2}=0.98, \mathrm{p}=0.32$; fungi fixed

517 feeding guild term: $\left.d f=1, \chi^{2}=0.09, \mathrm{p}=0.76\right)$. However, gut microbial community composition

518 did differ between the feeding guilds (bacteria: permanova, $F=13.2, R^{2}=0.040, p=0.001$, Figure

519 3a; fungi: permanova, $\mathrm{F}=7.4, \mathrm{R}^{2}=0.038, \mathrm{p}=0.001$, Figure $3 \mathrm{~b}$ ). Host feeding guild explained $4 \%$

520 of the variation in both bacterial and fungal community compositions.

521 Despite substantial inter-individual and inter-specific variation in microbial community

522 composition (described below), average relative abundances of 10 of the 20 most abundant

523 bacteria (Figure 4) and fungi (Figure 5) systematically differed between the feeding guilds. Of 
524 the bacteria, Swaminanthia/Asaia sp., Bartonella sp. and a strain of Commensalibacter intestini

were at higher relative abundance in nectivores than frugivores. Another strain of

Commensalibacter intestini, Wolbachia sp., a Porphyromonadaceae species, Gilliamella/Orbus

sp., Orbus sp. and Acetobacter sp. were at higher relative abundance in frugivores than

nectivores. Of the fungi, an unidentified fungus (OTU 7), a Letiomycetes species and a

529 Pleosporales species were more abundant in nectivores, while an unidentified ascomycete (OTU

20), two strains of Kazachstania exigua, Hanseniaspora guilliermondii, H. uvarum, H. opuntiae,

531 and Pichia fermentans associated more strongly with frugivores.

\section{2b. Variation among host species}

534 Observed OTU richness differed among butterfly species (Supplementary Figure 2; bacteria

535 random host species intercept term: $d f=1, \chi^{2}=43.3, \mathrm{p}<0.001$; fungi random host species

536 intercept term: $\left.d f=1, \chi^{2}=52.3, \mathrm{p}<0.001\right)$. Gut microbial community composition also differed

537 among host species (bacteria: permanova host species term, $d f=35, \mathrm{~F}=2.3, \mathrm{R}^{2}=0.244, \mathrm{p}<0.001$;

538 fungi: permanova host species term, $\left.\mathrm{F}=2.3, \mathrm{R}^{2}=0.319, \mathrm{p}<0.001\right)$. Host species explained $24 \%$

539 and $32 \%$ of the variation in bacterial and fungal community composition, respectively. Gut flora

540 were more similar within than between species (Figure 6).

\section{2c. Variation among individuals}

543 The vast majority of variation in community composition was expressed among individuals:

544 After accounting for species- and guild-level differences, residual variation in community

545 composition was $71.7 \%$ for bacteria and $64.4 \%$ for fungi (permanova). On average, a pair of

546 butterflies shared 23\% (IQR 13\%-31\%) of their bacterial OTUs and 10\% (IQR 0\%-16\%) of their 
547 fungal OTUs. No OTU was present in all butterfly individuals. Each bacterial OTU was found in

548 a mean of 9 individuals (IQR: 1-6) and each fungal OTU was found in a mean of 4 individuals

549 (IQR: 1-3).

\section{Comparison of the gut flora to food microbial communities}

We sequenced the bacterial flora of 87 food samples, 83 of which were retained after

rarefaction, and the fungal flora of 75 foods, 66 of which were retained after rarefaction (Table 1;

554 Supplementary Table 2). After sequence processing and quality filtering we obtained 7.7 million

555 bacterial and 3.2 fungal sequences from potential food sources (fruits, nectars, and trap baits).

556 After rarefying, a total of 1205 bacterial OTUs and 529 fungal OTUs were observed across all

557 the food samples. (Again note that numbers of bacterial and fungal OTUs cannot be compared

558 due to differences in rarefaction depth.)

The microbial communities in wild fruit juice, the fermented trap baits, and wild nectars

560 were distinct from each other and from butterfly gut communities (Figure 7; Figure 8). In fact,

561 butterfly gut communities were highly dissimilar to the microbial communities on their food

562 sources. For example, frugivores and wild fruits shared 7\% of their bacterial OTUs on average

563 (IQR 3\%-9\%), and the median Bray-Curtis dissimilarity between their bacterial communities

564 was 0.91 (Figure 8a). Despite these pronounced differences, frugivores' gut bacteria more

565 closely resembled the microbial composition of the trap baits than nectivores' gut bacteria did,

566 and similarly, nectivores' gut communities were more similar to nectar bacteria than frugivores'

567 gut flora were (Figure 8a). Frugivores' and nectivores' bacterial communities, however, were

568 equivalently distinct from those inhabiting the surfaces of wild fruits (Figure 8a).

$569 \quad$ Fungal communities in frugivore guts more closely resembled those of wild fruits and 
trap baits than did those of nectivore guts, as expected (Figure $8 \mathrm{~b}$ ). Surprisingly, frugivores' gut communities were (Figure $8 \mathrm{~b}$ ), though the effect size was extremely small. however, accounted for a comparatively small $24 \%$ to $40 \%$ of butterfly gut fungal reads.

\section{Functional abilities: gut microbial community catabolism}

580 We measured the catabolic profiles of 248 gut communities via culture-based assays. Gut

581 communities digested an average of 51 substrates (IQR: 41-63) out of 71 total. As two

582 communities diverged in bacterial species composition, their catabolic profiles also became more

583 different; however, the effect size was very small (Mantel test: $r=0.067, p=0.013$ ). Catabolism

584 was not correlated with fungal community composition (Mantel test: $\mathrm{p}=0.50$ ).

Across the entire dataset, host diet explained just 3\% of the total variation in community

catabolic profile (permanova on Euclidean distances between catabolic profiles; $d f=1, \mathrm{~F}=7.6$,

$\left.\mathrm{R}^{2}=0.180, \mathrm{p}=0.001\right)$. After accounting for diet- and species-level differences, residual variation

589 among individuals in catabolic profile was $79.1 \%$.

591 that not all catabolic functions are relevant for bacteria in butterfly guts. To investigate diet- 
593 diet, substrate identity, and substrate class, controlling for butterfly identity and number of $16 \mathrm{~S}$

594 rRNA copies in the butterfly. We also tested an alternate random effects structure, nesting

595 butterfly individuals within butterfly species, but this did not improve model fit (anova: $d f=1, \chi^{2}$

$596=0.258 \mathrm{p}=0.61)$. Catabolism of several individual substrates differed markedly between the

597 feeding guilds (feeding guild by substrate interaction term: $d f=70, \chi^{2}=428.9 \mathrm{p}<<0.001$ ).

598 Frugivorous gut flora catabolized 15 substrates more actively than nectivorous gut communities

599 did, with the most marked difference being in catabolism of D-serine, saccharic acid, mucic acid,

600 L-serine and lactic acid (Supplementary Table 5). Nectivorous gut flora digested 10 substrates

601 more actively, with the most extreme differences in catabolism of mannitol, sucrose, glucose,

602 fructose, and maltose (Supplementary Table 5). Entire classes of nutrients were also catabolized

603 differently between the guilds (feeding guild by substrate class interaction term: $d f=6, \chi^{2}=61.7$,

$604 \mathrm{p}<<0.001)$. Specifically, nectivores' gut flora catabolized sugars and sugar alcohols more

605 actively than frugivorous gut flora, while frugivores' gut flora were more successful at digesting

606 amino acids, carboxylic acids, and dicarboxylic acids (Figure 9; Supplementary Table 6).

607 Total number of $16 \mathrm{~S}$ rRNA copies was not significantly correlated with catabolism $(\mathrm{p}=$

6080.09 for the model of substrate identity and $p=0.07$ for the model of substrate class), suggesting

609 that our standardization procedure adequately controlled for any initial differences in cell

610 inoculation densities.

\section{Discussion}

613 We evaluated how microbial community structure and catabolic function varied among

614 host feeding guilds, species and individuals within a community of over 50 species of

615 Neotropical butterflies. On average, 30 bacterial OTUs and 21 fungal OTUs were detected per 
616 gut. Although the bacterial and fungal communities in adult butterfly guts varied substantially

617 among individual hosts, they did differentiate among host species and between host feeding

618 guilds. Gut community composition varied less between host feeding guilds than among host

619 species, suggesting that other non-dietary aspects of host biology play a large role in structuring

620 the butterfly gut flora. Gut communities were distinct in composition from the microbial flora

621 found in butterfly foods, indicating that the adult butterfly gut environment strongly filters

622 potential colonists. Despite high variability in the gut microbial community, host dietary guild

623 was nevertheless associated with consistent changes in both the relative abundances of dominant

624 microbes and the catabolic capacities of the gut flora. Frugivorous gut communities were better

625 at digesting amino acids and carboxylic acids, while nectivorous gut flora outperformed

626 frugivorous communities in catabolism of sugars and sugar alcohols. These catabolic patterns are

627 congruent with the relative nutritional makeup of butterfly foods (Ravenscraft and Boggs 2016),

628 which suggests that gut flora specialize in digestion of compounds abundant in the host's diet.

$630 \quad$ 1. Microbes present in the guts of adult butterflies

631 1a. Bacteria

632 The composition of the butterfly's bacterial flora is broadly similar to that of other

633 insects: at the phylum-level, Proteobacteria comprised $68 \%$ and Firmicutes $12 \%$ of all reads

634 while in comparison, a meta-analysis of the insect gut flora reported that $57 \%$ were

635 Proteobacteria and 22\% were Firmicutes (Colman et al 2012). At finer taxonomic resolution (see

636 Table 2), the most abundant bacterial OTUs were mostly known gut colonists, particularly those

637 associated with sugar-rich diets, and OTUs related to insect parasites and pathogens. (Individual

638 OTUs are discussed in more detail below.) Total bacterial load varied among species, but there 
were no consistent differences between the feeding guilds after controlling for differences in size among individual hosts. Larger butterfly individuals hosted greater numbers of bacteria. This

641 conforms previous work which demonstrated that total microbial load scales with host size (Kieft and Simmons 2015).

644 Heliconius erato butterflies in Panama (Hammer et al 2014). The genera Orbus, Enterobacter,

645 Asaia, Enterococcus, Lactococcus and Commensalibacter were abundant in both studies, and

646 Orbus has also been isolated from the gut of the butterfly Sasakia charonda in South Korea (Kim

647 et al 2013). This suggests that adult butterflies do form consistent associations with some gut

648 microbes. What drives these associations is uncertain. Butterflies might maintain specific

649 relationships with some microbes via mechanisms such as vertical transmission. Gut flora can be

650 transmitted from mother to offspring via internal migration of gut microbes into the eggs, as

651 observed in the moth Galleria mellonella, or via the smearing of gut microbes on egg surfaces,

652 which has been hypothesized to occur in tobacco hornworm (Manduca sexta) (Brinkmann et al

653 2008; Freitak et al 2014). The relative frequency of such inter-generational transmission is

654 unclear, however, because the majority of the larval gut flora are purged during metamorphosis

655 (Kingsley 1972; Hammer et al 2014; but see Johnston and Rolff 2015). Other members of the gut

656 flora may be common in both butterflies and other insects because they are generally adapted to

657 the insect gut environment (e.g. Chouaia et al 2012) and opportunistically colonize many species.

658 Many of the most abundant genera we detected may fall into this category.

659

1b. Gut fungi were primarily yeasts and common plant associates. 
662 all fungal sequences belonged to the order Saccharomycetales, the "budding yeasts" or "true

663 yeasts.” These single-celled fungi are often associated with sugar-rich environments and

664 decaying vegetable matter. Most of the remaining OTUs with reliable taxonomic assignments

665 were likely plant associates or pathogens. Studies characterizing the gut flora often focus on

666 bacteria and overlook fungi, but available evidence suggests that some insect guts host a rich

667 diversity of fungi, especially yeasts (Suh et al 2005). Some of these fungi may play an active role

668 in the gut, while others may simply use insects as a means of dispersal (Blackwell and Jones

669 1997; Starmer and Lachance 2011).

670

671 2. Variation in gut microbial community composition between host dietary guilds, among

672 species, and among individuals

673

674 2a. A smaller degree of variation was attributable to differences between feeding guilds.

675 Frugivores and nectivores did not differ in total bacterial load nor in bacterial or fungal

676 species richness, but did differ in the composition of their microbial flora. The degree of

677 variation explained by dietary guild was relatively small (about $4 \%$ for bacteria and fungi),

678 however it was comparable to that observed in many studies of microbial communities and is

679 likely biologically relevant: relative abundances of half of the most common OTUs differed

680 systematically between the feeding guilds. In general, the fungi more abundant in nectivores

681 were closely related to plant pathogens, while the fungi more abundant in frugivores were yeasts

682 associated with fruit decay, suggesting that this variation likely derived from exposure to

683 different microbial source pools. In contrast, there was no pattern in the types of bacteria that

684 differed in abundance between frugivores and nectivores. 
2b. A moderate degree of variation was attributable to differences among host species.

About $24 \%$ and $32 \%$ percent of the variation in bacterial and fungal OTU compositions, respectively, was attributed to differences among host species. Total bacterial load and bacterial and fungal OTU richness also differed among butterfly species. Interspecies variation in the butterfly gut flora could result from behavioral differences that expose butterflies to different microbial pools. Such species traits could include habitat preferences. For example, species that favor the forest understory could be exposed to more soil and wood decay fungi than those that prefer the canopy. Additionally, many, but not all species drink from mud, dung, or carrion, in a behavior known as "puddling” (Adler and Pearson 1982; Sculley and Boggs 1996), and variation in these auxiliary feeding substrates could introduce different microbes to the gut. Differences could also arise from variation in gut chemistry: for example, the guts of larger butterflies might be more anoxic, or there could be interspecies differences in gut $\mathrm{pH}$.

At least some of these factors could exhibit evolutionary signal in butterflies, resulting in correlation between the butterfly phylogeny and gut community composition. Alternatively or additionaly, vertical transmission of a fraction of the gut community from parent to offspring could result in parallel divergence of microbiota and hosts. The potential for such evolutionary patterns to contribute to interspecies differences in gut community composition is beyond the scope of the current study, but will be addressed in a future paper (Ravenscraft et al, in prep). 2c. Variation in microbial community composition among individual butterflies is high, but not unusual.

There was a great deal of variation among individual butterflies in microbial community composition: the most prevalent bacterial and fungal OTUs were detected in only $83 \%$ and of 
$45 \%$ butterflies, respectively, and half of all OTUs were found in only one individual. Although this degree of variation may seem extreme, it is comparable to that observed in other animals:

711 human colon microbiota were 34\% similar among individuals in one study (Green et al 2006),

712 and in another, no OTUs were universally shared and 79\% were found in only a single individual

713 (Tap et al 2009). A study of mammalian gut flora found that, on average, $56 \%$ of OTUs in an

714 individual mammal were unique to that individual (Ley et al 2008a). It has been proposed that

715 the high degree of variation observed in gut communities is the result of functional redundancy

716 at the OTU level: many microbes may require similar gut environmental conditions and/or serve

717 similar functional roles, leading to communities that differ in OTU membership but not in

718 overall function (Ley et al 2006; Peay et al 2016). However, it is also possible that variation in

719 membership does lead to significant variation in function (Peay et al 2016) and that the butterfly

720 gut flora do not play a consistent role across individuals.

2d. The effect sizes of variables related to gut community composition are rarely reported or

723 compared.

We had expected that a butterfly's feeding guild would explain a larger amount of

725 variation in gut community membership than host species identity, but we found the opposite.

726 The relative importance of dietary guild as a determinant of gut community composition has

727 been obscured in the literature by a trend of reporting only the significance (p-value) and not the

728 strength (either the effect size or R-squared) of these relationships. Diet has been shown to

729 significantly affect the community composition of the gut flora in many systems, including

730 mammals (Ley et al 2008a), fish (Sullam et al 2012), and insects (Colman et al 2012), but the

731 strength of this correlation was only reported for the last case. In a broad survey of the insect gut 
732 flora, feeding guild (e.g. herbivore, omnivore, detritivore, pollenivore) explained 23\% of

733 variation in the gut flora. The comparatively small amount of variation which diet explains in the

734 butterfly gut flora could be due in part to the broadly similar nature of butterfly foods (both being 735 sugary liquids).

737 3. Comparison of gut flora to food microbial communities.

738 3a. Butterfly gut communities were highly dissimilar to fruit and nectar communities, but

739 generally shared more OTUs with the diet of their host than the diet of the other feeding guild.

740 Host diet may shape the gut flora by serving as a source pool of microbes. Since it is

741 likely that adult butterflies acquire a substantial fraction of their gut flora from the environment

742 after emergence from the pupa (Hammer et al 2014), the microbial composition of adult butterfly

743 foods could be especially relevant. We asked whether the gut flora of Neotropical butterflies

744 might derive from their foods, and tested whether differences between frugivore and nectivore

745 gut communities were driven, in part, by differences in the microbes present in fruits and nectars.

746 Butterfly gut communities were more dissimilar to food microbial communities than we had

747 anticipated, with median percentage OTUs shared between a gut community and the microbes

748 residing in its host's food ranging from 5 to $16 \%$ for bacteria and 0 to $14 \%$ for fungi. However,

749 gut flora were generally more similar to the community on their host's food than to microbes on

750 the food of the other feeding guild. This suggests that a limited number of food-derived bacteria

751 and fungi either pass through or colonize the gut. Indeed, comparison of frugivores caught with

752 aerial nets to those caught via baited traps indicated that the yeast Kazachstania exigua was

753 transferred from the fermented baits into trapped butterflies' guts. 
The high degree of differentiation between gut and food communities was surprising because the guts of many nonsocial insects do seem to be colonized by environmental bacteria (Ley et al 2008b; Boissière et al 2012). Indeed, prior studies of lepidopterans have found that lab-reared larvae have a depauperate gut community compared to wild individuals, suggesting that the larval gut is populated by microbes acquired from the environment (Xiang et al 2006; Pinto-Tomás and Sittenfeld 2011; Belda et al 2011). Although the adult butterfly gut probably is colonized at least in part by environmental microbes - as suggested by the high percentage of bacterial reads derived from OTUs also found in butterfly foods - the vast majority of ingested microbial species may fail to establish residence. The fact that the majority of the OTUs in butterfly foods were not detected in adult guts emphasizes the strength of the filter the gut exerts on potential microbial colonists, even in non-social hosts such as butterflies. Gut chemistry likely filters potential colonists: most of the microbes in the gut (excluding transients) will belong to the subset that can tolerate the gut's $\mathrm{pH}$ and oxygenation conditions. Competition or priority effects from initial gut residents (those carried over from the larval host or established from early adult meals) could also prevent food-derived microbes from successful colonization. Both areas are fruitful avenues for future research aimed at understanding the community assembly of animal gut flora.

\section{3b. Known habitat requirements of dominant gut microbes suggest that the adult butterfly gut is} acidic and potentially aerobic.

The environmental requirements of the gut flora can help us infer chemical conditions in the adult butterfly gut, which can be difficult to measure using traditional means. Acetic acid bacteria (Acetobacteraceae) are obligately aerobic (Komagata et al 2014). The presence of many 
members of the Acetobacteraceae in the adult gut suggests that it is not entirely anoxic; oxygen may reach the outer layer of the gut via diffusion from gas in the tracheoles. Acetobacteraceae also prefer acidic conditions, as do the yeasts Hanseniaspora, Candida and Kazachstania (Rosa and Peter 2006; Komagata et al 2014). We could find no information about the $\mathrm{pH}$ of the adult

781 butterfly's gut, but in adults of the moth Manduca sexta it is between 5 and 6 (T. Hammer,

782 unpublished data). The larval gut is often alkaline, ranging from close to neutral pH to over 12

783 (Gross et al 2008), but the prevalence of acetic acid bacteria and acid-tolerant yeasts in our data 784 suggests that the adult gut is in fact acidic across most, if not all, butterfly species.

4. Functional potential of the butterfly gut flora.

4a. The catabolic potential of the gut flora varies with host diet. acids compared to nectivores' gut flora, and decreased catabolism of sugars and sugar alcohols.

791 These differences in function likely result from differences in the chemical composition of the 792 diet. Nectars are 90\% sugar by dry weight (Luttge 1977). Fruit generally contains more nitrogen

793 than nectar: at our field site, the juice of rotting Dipteryx oleifera, the dominant food available to 794 frugivorous butterflies during portions of the year, contains 33 times more essential amino acids and 19 times more non-essential amino acids per unit sugar than the flower nectars fed upon by

796 butterflies (Ravenscraft and Boggs 2016). Frugivorous butterflies therefore provide a

797 comparatively nitrogen-rich gut environment that appears to support microbes capable of

798 catabolizing amino acids, while nectivores provide a more sugar-rich environment that favors

799 microbes specialized on sugar catabolism. 
A 96-well plate is a very different environment from a butterfly gut, therefore we cannot

801 conclude that the functions we observed necessarily operate in vivo. However, our results

802 indicate that host species and host diet are related not only to gut community composition, but

803 also the functional capacities of the gut flora, and they suggest that the chemical makeup of the

804 diet selects for microbes that are specialized to digest that diet.

805

806 5. Butterfly gut flora include potential mutualists, commensals and pathogens.

807 A full understanding of the microbial gut community ultimately requires specific knowledge of

808 each of its members. From what is known about the microbial genera present in the butterfly gut,

809 we can begin to speculate whether they may be beneficial, commensal, or detrimental to butterfly

810 hosts.

$812 \quad 5$ a. Bacteria

813 The genera Enterobacter, Enterococcus, and Lactococcus are commonly present in the

814 digestive tracts of a wide range of animals, from mammals to insects, and likely participate in

815 mutualistic or commensal relationships with their hosts (e.g. Robinson et al 2010; Engel and

816 Moran 2013; Delsuc et al 2014). Acetobacter, Asaia, and Pantoea are frequent residents of insect

817 guts (Crotti et al 2010; Engel and Moran 2013) and have been shown to directly benefit some

818 hosts. For example, Acetobacter pomorum and Asaia promote dipteran larval development,

819 probably through nutrient supplementation (Shin et al 2011; Mitraka et al 2013). Pantoea

820 agglomerans helps the swarming locust, Schistocerca gregaria, synthesize aggregation

821 pheromone (Dillon et al 2002). What these species may do for butterflies is currently unknown. 
We also detected several genera that might be detrimental to butterflies. Serratia species are often insect pathogens (Grimont and Grimont 2006). The genus Bartonella contains

824 opportunistic animal pathogens that can infect insects and mammals. Many are vectored by

825 insects — primarily flies (Minnick and Anderson 2000) — but the genus has also been detected in

826 the guts of other non-biting insects including honey bees, ants, and carrion beetles (Jeyaprakash

827 et al 2003; Stoll et al 2007; Kaltenpoth and Steiger 2014). Spiroplasma and Wolbachia are well-

828 known as reproductive parasites of insects; some kill males to promote their own spread via the

829 female line. Spiroplasma are known to colonize the gut lumen. Wolbachia are generally

830 associated with the reproductive organs, but have also been found in insect guts (Frost et al 2014;

831 Berasategui et al 2016). We dissected out the guts of the butterflies in our study, but we were not

832 always able to completely remove attached fat, Malphigian tubules, and reproductive tissue.

833 Although we are confident that most of the OTUs reported here were gut-derived, the origin of

834 the Wolbachia in our study is uncertain.

Although several of the most abundant bacterial genera we detected are often assumed to

836 be harmful to their hosts and could well be detrimental to butterflies, evidence suggests these

837 genera can sometimes be beneficial. Spiroplasma and Wolbachia can both protect their hosts

838 from pathogens or parasites (e.g. Hedges et al 2008; Jaenike et al 2010) and Wolbachia has been

839 shown to engage in nutritional symbioses, providing B vitamins to bedbugs (Hosokawa et al

840 2010). The high prevalence and vertical transmission of Bartonella in several fly species suggest

841 that this genus could also be beneficial to some insect hosts (Halos et al 2004). Overall,

842 butterflies host both potential mutualists and pathogens, but the balance seems shifted towards

843 more beneficial than detrimental bacteria. 
The data that do exist regarding the community composition of the adult butterfly gut

845 flora are largely congruent with our findings. Enterobacter, Enterococcus, Lactococcus,

846 Commensalibacter and Orbus were dominant members of the adult butterfly gut flora in both our

847 data and in a study of the butterfly Heliconius erato in Panama (Hammer et al 2014).

848 Commensalibacter has also been detected in Drosophila guts, where it may protect the flies from

849 infection by pathogens (Roh et al 2008; Ryu et al 2008). It may play a beneficial role in

850 butterflies, as well: in a related study, we found that abundance of Commensalibacter was

851 positively correlated with increased adult lifespan in the temperate butterfly Speyeria mormonia

852 (Ravenscraft et al, in review). Little is known about the biology of Orbus, but the genus or its

853 close relatives have been detected in the guts of flies (Chandler et al 2011), bees (Kwong and

854 Moran 2013), and geographically disparate butterflies including Speyeria mormonia in Colorado

855 and Sasakia charonda in South Korea (Kim et al 2013). The apparently widespread associations

856 between butterflies and bacteria in the genera Commensalibacter and Orbus suggest that these

857 microbes may have a generalized relationship with lepidopterans or, more broadly, with sugar-

858 feeding insects.

5b. Fungi

Most of the fungal inhabitants of butterfly guts were saccharomycete yeasts. Members of

862 the genus Hanseniaspora are early colonizers of decaying fruits and are also commonly isolated

863 from drosophilid flies. They are dispersed by the flies, which also use them as a food source

864 (Kurtzman et al 2011). The genera Candida and Pichia are globally widespread and present in

865 many different environments. Some are pathogenic, but many are animal commensals or 
867 invertebrates including humans, gorillas, beetles, and larval lepidopterans (Rosa et al 1992; Suh

868 et al 2005; Marchesi 2010; Dematheis et al 2012; Hamad et al 2014). All reported isolates of

869 Candida quercitrusa, the species most closely related to the OTUs in our dataset, derive from

870 flowers or insects (Kurtzman et al 2011). Not much is known about the ecology of Kazachstania

871 exigua, but most strains have been isolated from human food products (Kurtzman et al 2011).

872 While Kazachstania was present at artificially high abundance in our raw data due to its

873 dominance in the trap baits, it was also detected on wild fruits and in the guts of butterflies that

874 had not fed on trap baits, suggesting that it does naturally occur in butterfly guts at low

875 abundance.

Many of the abundant fungal OTUs outside of the Saccharomycetales were too divergent

from known species' sequences to identify them with fine taxonomic resolution. One OTU was a

879 Pleosporales, which predominantly consists of plant-decaying fungi, though some species

880 associate with live plants (Zhang et al 2009). These fungi may have arrived in the adult gut via

881 spores in fruits and nectars, or they could have colonized the larval gut via ingestion of leaf

882 tissue and then persisted through pupation. In summary, the fungal flora of the butterfly gut

883 included many sugar-loving yeasts and plant associates, some of which may pass through insect

884 guts as accidental vagrants or hitchhikers, as well as potential insect gut-associated commensals

885 or mutualists.

886

887 Conclusions

Gut microbiota are a ubiquitous feature of animal life, yet we still understand little about 
butterflies as a study system to partition variation in gut community membership at the levels of

891 the host individual, species, and feeding guild; to compare the microbial species in the gut to

892 those in host foods; and to investigate the catabolic potential of the gut flora. All samples were

893 collected from a single site, thus eliminating the potential for large-scale geographic variation

894 that is often present in, and can potentially confound, studies of similar scale.

895 We found that the majority of variation in the butterfly gut flora is expressed at the level

896 of the individual host, followed by host species, with the least amount of variation explained by

897 dietary guild. The consistency of these patterns across both bacterial and fungal communities

898 lends support to the observed pattern. The pattern itself demonstrates the need for future work to

899 expand from the current focus on broad diet categories and host taxonomy to additional, species-

900 level or individual-level traits - for example, gut chemistry — in order to better understand the

901 causes and consequences of the gut community structure in nature.

902 Butterfly gut flora had little resemblance to food microbial communities, suggesting that

903 conditions in the gut exert a strong ecological filter on colonists. The initial colonists of the

904 butterfly gut may be derived largely from butterfly foods, but once established, the butterfly gut

905 community appears to be resistant to perturbation via ingestion of microbes. However, foods do

906 influence the gut flora via their chemical composition: culture-based assays suggested that the

907 chemical composition of the diet selects for gut microbes that are good at digesting common

908 chemical components of that diet.

909 Butterfly guts host a rich community of bacteria, as well as yeasts, which are woefully

910 understudied in contemporary analyses of the gut flora. By characterizing variation of the gut

911 microbiota within and among butterfly species we have laid the foundation for a mechanistic

912 understanding of how this hidden symbiosis affects and is affected by its host. 


\section{Acknowledgments}

915 Sincere thanks to Luke Frishkoff, Meredith Blackwell, and Tadashi Fukami’s 2013 Ecological

916 Statistics class for insightful discussion and feedback. Jon Sanders provided the 16s rRNA

917 standard for our qPCR standard curve. Carlos de la Rosa, Ronald Vargas, Bernal Matarrita

918 Carranza, Danilo Brenes Madrigal and the staffs of OTS and La Selva Biological Station offered

919 invaluable logistical assistance and support in the field. This work was supported by an NSF

920 Graduate Research Fellowship, a Stanford Center for Computational, Evolutionary and Human

921 Genomics Graduate Fellowship, and an NIH PERT postdoctoral fellowship to AR, funds from

922 Stanford University, and grants from Stanford University’s Biology Department, Center for

923 Latin American Studies, and Biosciences Office of Graduate Education.

925 Data Accessibility

926 Raw Illumina sequences will be made available in the NCBI Short Read Archive. All other data

927 (e.g. the OTU table, bacterial abundances calculated from qPCR, butterfly fecundity, egg

928 chemical composition, etc) will be uploaded to Dryad.

929 


\section{Literature Cited}

931 Adler P, Pearson D (1982) Why do male butterflies visit mud puddles? Can J Zool 60:322-325.

932 Anderson MJ (2001) A new method for non-parametric multivariate analysis of variance. Austral Ecol 26:32-46. doi: 10.1111/j.1442-9993.2001.01070.pp.x

934 Anderson MJ, Walsh DCI (2013) PERMANOVA, ANOSIM, and the Mantel test in the face of heterogeneous 935 dispersions: What null hypothesis are you testing? Ecol Monogr 83:557-574. doi: 10.1890/12-2010.1

Beasley DE, Koltz AM, Lambert JE, et al (2015) The evolution of stomach acidity and its relevance to the human microbiome. PLoS One 10:1-12. doi: 10.1371/journal.pone.0134116

Belda E, Pedrola L, Peretó J (2011) Microbial diversity in the midguts of field and lab-reared populations of the European Corn Borer Ostrinia nubilalis. PLoS One 6:e21751. doi: 10.1371/journal.pone.0021751

Benjamini Y, Hochberg Y (1995) Controlling the false discovery rate: A practical and powerful approach to multiple testing. J R Stat Soc Ser B 57:289-300. doi: 10.2307/2346101

Berasategui A, Axelsson K, Norlander G, et al (2016) The gut microbiota of the pine weevil (Hylobius abietis, Coleoptera: Curculionidae) is similar across Europe and resembles that of other confer-feeding beetles. Mol Ecol. doi: 10.1111/mec.13702

Bergmann GT, Bates ST, Eilers KG, et al (2011) The under-recognized dominance of Verrucomicrobia in soil bacterial communities. Soil Biol Biochem 43:1450-1455. doi: 10.1016/j.soilbio.2011.03.012

Blackwell M, Jones K (1997) Taxonomic diversity and interactions of insect-associated ascomycetes. Biodivers Conserv 6:689-699. doi: 10.1023/A:1018366203181

Boissière A, Tchioffo MT, Bachar D, et al (2012) Midgut microbiota of the malaria mosquito vector Anopheles gambiae and interactions with Plasmodium falciparum infection. PLoS Pathog 8:e1002742. doi:

Brinkmann N, Martens R, Tebbe CC (2008) Origin and diversity of metabolically active gut bacteria from laboratory-bred larvae of Manduca sexta (Sphingidae, Lepidoptera, Insecta). Appl Environ Microbiol 74:7189-7196. doi: 10.1128/AEM.01464-08

Broderick NA, Raffa KF, Goodman RM, Handelsman J (2004) Census of the bacterial community of the gypsy 
Caporaso JG, Lauber CL, Walters WA, et al (2012) Ultra-high-throughput microbial community analysis on the Illumina HiSeq and MiSeq platforms. ISME J 6:1621-1624. doi: 10.1038/ismej.2012.8

960 Chandler JA, Lang J, Bhatnagar S, et al (2011) Bacterial communities of diverse Drosophila species: Ecological 961 context of a host-microbe model system. PLoS Genet 7:e1002272. doi: 10.1371/journal.pgen.1002272

962 Chouaia B, Rossi P, Epis S, et al (2012) Delayed larval development in Anopheles mosquitoes deprived of Asaia

963 bacterial symbionts. BMC Microbiol 12:S2. doi: 10.1186/1471-2180-12-S1-S2

964 Colman DR, Toolson EC, Takacs-Vesbach CD (2012) Do diet and taxonomy influence insect gut bacterial communities? Mol Ecol 21:5124-5137. doi: 10.1111/j.1365-294X.2012.05752.x

Crotti E, Rizzi A, Chouaia B, et al (2010) Acetic acid bacteria, newly emerging symbionts of insects. Appl Environ Microbiol 76:6963-6970. doi: 10.1128/AEM.01336-10

Daily GC, Ehrlich PR (1995) Preservation of biodiversity in small rainforest patches: rapid evaluations using butterfly trapping. Biodivers Conserv 4:35-55. doi: 10.1007/BF00115313

970 Delsuc F, Metcalf JL, Wegener Parfrey L, et al (2014) Convergence of gut microbiomes in myrmecophagous mammals. Mol Ecol 23:1301-1317. doi: 10.1111/mec.12501

Dematheis F, Kurtz B, Vidal S, Smalla K (2012) Microbial communities associated with the larval gut and eggs of the western corn rootworm. PLoS One 7:e44685. doi: 10.1371/journal.pone.0044685

Deshpande V, Wang Q, Greenfield P, et al (2016) Fungal identification using a Bayesian classifier and the Warcup training set of internal transcribed spacer sequences. Mycologia 108:1-5. doi: 10.3852/14-293

Dillon RJ, Dillon VM (2004) The gut bacteria of insects: nonpathogenic interactions. Annu Rev Entomol 49:71-92. doi: 10.1146/annurev.ento.49.061802.123416

Dillon RJ, Vennard CT, Charnley AK (2002) A note: Gut bacteria produce components of a locust cohesion pheromone. J Appl Microbiol 92:759-763. doi: 10.1046/j.1365-2672.2002.01581.x

Edgar RC (2013) UPARSE: highly accurate OTU sequences from microbial amplicon reads. Nat Methods 10:9968. doi: $10.1038 /$ nmeth. 2604

Engel P, Moran NA (2013) The gut microbiota of insects - diversity in structure and function. FEMS Microbiol Rev 37:699-735. doi: 10.1111/1574-6976.12025 
Frost CL, Pollock SW, Smith JE, Hughes WOH (2014) Wolbachia in the flesh: Symbiont intensities in germ-line and somatic tissues challenge the conventional view of Wolbachia transmission routes. PLoS One 9:e95122. doi: 10.1371/journal.pone.0095122

Gardes M, Bruns TD (1993) ITS primers with enhanced specificity for basidiomycetes- application to the identification of mycorrhizae and rusts. Mol Ecol 2:113-118. doi: Doi 10.1111/J.1365-294x.1993.Tb00005.X

Garland JL, Mills AL, Young JS (2001) Relative effectiveness of kinetic analysis vs single point readings for classifying environmental samples based on community-level physiological profiles (CLPP). Soil Biol Biochem 33:1059-66.

Gilbert JA, Jansson JK, Knight R (2014) The Earth Microbiome project: successes and aspirations. BMC Biol 12:69. doi: 10.1186/s12915-014-0069-1

Gordon GL, Phillips MW (1998) The role of anaerobic gut fungi in ruminants. Nutr Res Rev 11:133-68. doi: 10.1079/NRR19980009

Green GL, Brostoff J, Hudspith B, et al (2006) Molecular characterization of the bacteria adherent to human colorectal mucosa. J Appl Microbiol 100:460-469. doi: 10.1111/j.1365-2672.2005.02783.x

Grimont F, Grimont PAD (2006) The Genus Serratia. In: Balows A, Trüper H, Dworkin M, et al (eds) Prokaryotes. Springer-Verlag, New York, pp 219-244

Gross EM, Brune A, Walenciak O (2008) Gut pH, redox conditions and oxygen levels in an aquatic caterpillar: Potential effects on the fate of ingested tannins. J Insect Physiol 54:462-471. doi: 10.1016/j.jinsphys.2007.11.005

Hakim RS, Baldwin K, Smagghe G (2010) Regulation of midgut growth, development, and metamorphosis. Annu Rev Entomol 55:593-608. doi: 10.1146/annurev-ento-112408-085450

Halos L, Jamal T, Maillard R, et al (2004) Role of Hippoboscidae flies as potential vectors of Bartonella spp. infecting wild and domestic ruminants. Appl Environ Microbiol 70:6302-6305. doi: 10.1128/AEM.70.10.6302-6305.2004

Hamad I, Keita MB, Peeters M, et al (2014) Pathogenic eukaryotes in gut microbiota of western lowland gorillas as revealed by molecular survey. Sci Rep 4:1-9. doi: 10.1038/srep06417

Hammer TJ, Dickerson JC, Fierer N (2015) Evidence-based recommendations on storing and handling specimens for analyses of insect microbiota. PeerJ e1190. doi: 10.7717/peerj.1190 
1014 Hammer TJ, McMillan WO, Fierer N (2014) Metamorphosis of a butterfly-associated bacterial community. PLoS One 9:e86995. doi: 10.1371/journal.pone.0086995

1016 Hedges LM, Brownlie JC, O’Neill SL, Johnson KN (2008) Wolbachia and virus protection in insects. Science 1017 322:702. doi: $10.1126 /$ science. 1162418

1018 Hosokawa T, Koga R, Kikuchi Y, et al (2010) Wolbachia as a bacteriocyte-associated nutritional mutualist. Proc $1019 \quad$ Natl Acad Sci 107:769-774. doi: 10.1073/pnas.0911476107

1020 Jaenike J, Unckless R, Cockburn SN, et al (2010) Adaptation via symbiosis: recent spread of a Drosophila defensive $1021 \quad$ symbiont. Science 329:212-215. doi: $10.1126 /$ science. 1188235

1022 Jeyaprakash A, Hoy MA, Allsopp MH (2003) Bacterial diversity in worker adults of Apis mellifera capensis and 1023 Apis mellifera scutellata (Insecta: Hymenoptera) assessed using 16S rRNA sequences. J Invertebr Pathol 84:96-103. doi: 10.1016/j.jip.2003.08.007

Johnson KS, Barbehenn R V. (2000) Oxygen levels in the gut lumens of herbivorous insects. J Insect Physiol 46:897-903. doi: 10.1016/S0022-1910(99)00196-1

Johnston PR, Rolff J (2015) Host and symbiont jointly control gut microbiota during complete metamorphosis. PLoS Pathog 11:e1005246. doi: 10.1371/journal.ppat.1005246

Kaltenpoth M, Steiger S (2014) Unearthing carrion beetles' microbiome: Characterization of bacterial and fungal hindgut communities across the Silphidae. Mol Ecol 23:1251-1267. doi: 10.1111/mec.12469

Kieft TL, Simmons KA (2015) Allometry of animal-microbe interactions and global census of animal-associated microbes. Proc R Soc B Biol Sci 282:20150702. doi: 10.1098/rspb.2015.0702

1033 Kim JY, Lee J, Shin N-R, et al (2013) Orbus sasakiae sp. nov., a bacterium isolated from the gut of the butterfly Sasakia charonda, and emended description of the genus Orbus. Int J Syst Evol Microbiol 63:1766-1770. doi:

$1035 \quad 10.1099 /$ ijs.0.041871-0

1036 Kingsley V (1972) Persistence of intestinal bacteria in the developmental stages of the Monarch butterfly (Danaus 1037 plexippus). J Invertebr Pathol 20:51-58.

1038 Kirk P, Cannon P, Minter D, Stalpers J (eds) (2008) Dictionary of the Fungi, 10th edn. CABI, Wallingford, UK 1039 Komagata K, Iino T, Yamada Y (2014) The Family Acetobacteraceae. In: Rosenberg E, DeLong EF, Lory S, et al 1040 (eds) Prokaryotes Vol. 8 Alphaproteobacteria Betaproteobacteria, 4th edn. pp 3-78

1041 Kozich JJ, Westcott SL, Baxter NT, et al (2013) Development of a dual-index sequencing strategy and curation 

Microbiol 79:5112-5120. doi: 10.1128/AEM.01043-13

1044 Kurtzman CP, Fell JW, Boekhout T (eds) (2011) The Yeasts, A Taxonomic Study, Volume 2, 5th edn. Elsevier, San 1045 Diego, CA

1046 Kwong WK, Moran NA (2013) Cultivation and characterization of the gut symbionts of honey bees and bumble

1047 bees: Description of Snodgrassella alvi gen. nov., sp. nov., a member of the family Neisseriaceae of the 1048 betaproteobacteria, and Gilliamella apicola gen. nov., sp. Int J Syst Evol Microbiol 63:2008-2018. doi:

$1049 \quad 10.1099 /$ ijs.0.044875-0

1050 Lenth R V (2016) Least-Squares Means: The R Package 1smeans. J Stat Softw 69:1-33. doi: 10.18637/jss.v069.i01

1051 Ley RE, Hamady M, Lozupone C, et al (2008a) Evolution of mammals and their gut microbes. Science 320:16471052 1651. doi: $10.1126 /$ science. 1155725

1053 Ley RE, Lozupone CA, Hamady M, et al (2008b) Worlds within worlds: evolution of the vertebrate gut microbiota. $1054 \quad$ Nat Rev Microbiol 6:776-788. doi: 10.1038/nrmicro1978

1055 Ley RE, Peterson DA, Gordon JI (2006) Ecological and evolutionary forces shaping microbial diversity in the human intestine. Cell 124:837-848. doi: 10.1016/j.cell.2006.02.017

1057 Lundberg DS, Yourstone S, Mieczkowski P, et al (2013) Practical innovations for high-throughput amplicon 1058 sequencing. Nat Methods 10:999-1002. doi: 10.1038/nmeth.2634

1059 Luttge U (1977) Nectar composition and membrane transport of sugars and amino acids: a review on the present 1060 state of nectar research. Apidologie 8:305-319. doi: 10.1051/apido:19770402

1061 Marchesi JR (2010) Prokaryotic and eukaryotic diversity of the human gut. Adv. Appl. Microbiol., 1st edn. Elsevier Inc., pp 43-62

1063 Martin M (2011) Cutadapt removes adapter sequences from high-throughput sequencing reads. EMBnet.journal $1064 \quad$ 17:10. doi: $10.14806 /$ ej.17.1.200

1065 McDade LA, Bawa KS, Hespenheide HA, Hartshorn GS (1994) La Selva: ecology and natural history of a 1066 Neotropical rain forest. University of Chicago Press, Chicago

1067 McDonald D, Price MN, Goodrich J, et al (2012) An improved Greengenes taxonomy with explicit ranks for 1068 ecological and evolutionary analyses of bacteria and archaea. ISME J 6:610-618. doi: 10.1038/ismej.2011.139 1069 McFall-Ngai M (2007) Adaptive immunity: care for the community. Nature 445:153. doi: 10.1038/445153a 
McMurdie PJ, Holmes S (2013) Phyloseq: An R package for reproducible interactive analysis and graphics of microbiome census data. PLoS One 8:e61217. doi: 10.1371/journal.pone.0061217

1072 McNulty NP, Yatsunenko T, Hsiao A, et al (2011) The impact of a consortium of fermented milk strains on the gut 1073 microbiome of gnotobiotic mice and monozygotic twins. Sci Transl Med 3:1-14. doi: 10.1126/scitranslmed.3002701

Minnick MF, Anderson BE (2000) Bartonella interactions with host cells. In: Oelschlaeger TA, Hacker JH (eds) Subcell. Biochem. Bact. Invasion into Eukaryot. Cells. Springer, New York, NY, pp 97-118

Mitraka E, Stathopoulos S, Siden-Kiamos I, et al (2013) Asaia accelerates larval development of Anopheles gambiae. Pathog Glob Health 107:305-11. doi: 10.1179/2047773213Y.0000000106

1079 Muegge BD, Kuczynski J, Knights D, et al (2011) Diet drives convergence in gut microbiome functions across mammalian phylogeny and within humans. Science 332:970-4. doi: 10.1126/science.1198719

Nardi JB, Mackie RI, Dawson JO (2002) Could microbial symbionts of arthropod guts contribute significantly to 1082 nitrogen fixation in terrestrial ecosystems? J Insect Physiol 48:751-763.

1083 Oksanen J, Blanchet FG, Kindt R, et al (2015) vegan: Community Ecology Package. R package version 2.3-0.

Peay KG, Bruns TD, Kennedy PG, et al (2007) A strong species-area relationship for eukaryotic soil microbes: Island size matters for ectomycorrhizal fungi. Ecol Lett 10:470-480. doi: 10.1111/j.1461-0248.2007.01035.x

Peay KG, Kennedy PG, Talbot JM (2016) Dimensions of biodiversity in the Earth mycobiome. Nat Rev Microbiol 14:434-447. doi: 10.1038/nrmicro.2016.59

Pinto-Tomás A, Sittenfeld A (2011) Comparison of midgut bacterial diversity in tropical caterpillars (Lepidoptera: Saturniidae) fed on different diets. Environ Entomol 40:1111-1122.

R Core Team (2015) R: A language and environment for statistical computing. R Foundation for Statistical

1091 Computing, Vienna, Austria. URL http://www.R-project.org/.

1092 Ravenscraft A, Boggs CL (2016) Nutrient acquisition across a dietary shift: fruit feeding butterflies crave amino acids, nectivores seek salt. Oecologia 181:1-12. doi: 10.1007/s00442-015-3403-6

1094 Rawls JF, Mahowald MA, Ley RE, Gordon JI (2006) Reciprocal gut microbiota transplants from zebrafish and mice 1095 to germ-free recipients reveal host habitat selection. Cell 127:423-433. doi: 10.1016/j.cell.2006.08.043 microbial communities. Microbiol Mol Biol Rev 74:453-76. doi: 10.1128/MMBR.00014-10 
Robinson CJ, Schloss P, Ramos Y, et al (2010b) Robustness of the bacterial community in the cabbage white butterfly larval midgut. Microb Ecol 59:199-211. doi: 10.1007/s00248-009-9595-8

Roh SW, Nam Y-D, Chang H-W, et al (2008) Phylogenetic characterization of two novel commensal bacteria involved with innate immune homeostasis in Drosophila melanogaster. Appl Environ Microbiol 74:6171-

Rosa CA, Hagler AN, Mendonca-Hagler LCS, et al (1992) Clavispora opuntiae and other yeasts associated with the moth Sigelgaita sp. in the cactus Pilosocereus arrabidae of Rio de Janeiro, Brazil. Antonie Van Leeuwenhoek 62:267-272. doi: 10.1007/BF00572594

Rosa CA, Peter G (eds) (2006) Biodiversity and Ecophysiology of Yeasts. Springer-Verlag

Russell V, Dunn PE (1996) Antibacterial proteins in the midgut of Manduca sexta during metamorphosis. J Insect Physiol 42:65-71. doi: 10.1016/0022-1910(95)00083-6

Ryu JH, Kim SH, Lee HY, et al (2008) Innate immune homeostasis by the homeobox gene caudal and commensalgut mutualism in Drosophila. Science 319:777-782. doi: 10.1126/science.1149357

Salter SJ, Cox MJ, Turek EM, et al (2014) Reagent and laboratory contamination can critically impact sequence-

1113 Salzman NH, Hung K, Haribhai D, et al (2009) Enteric defensins are essential regulators of intestinal microbial 1114 ecology. Nat Immunol 11:76-82. doi: 10.1038/ni.1825

1115 Sculley CE, Boggs CL (1996) Mating systems and sexual division of foraging effort affect puddling behaviour by $1116 \quad$ butterflies. Ecol Entomol 21:193-197.

1117 Shin SC, Kim S-H, You H, et al (2011) Drosophila microbiome modulates host developmental and metabolic 1118 homeostasis via insulin signaling. Science 334:670-4. doi: 10.1126/science.1212782

1119 Smith DP, Peay KG (2014) Sequence depth, not PCR replication, improves ecological inference from next 1120 generation DNA sequencing. PLoS One 9:e90234. doi: 10.1371/journal.pone.0090234

1121 Solomon K V., Haitjema CH, Henske JK, et al (2016) Early-branching gut fungi possess a large, comprehensive 1122 array of biomass-degrading enzymes. Science 351:1192-1195. doi: 10.1126/science.aad1431

1123 Starmer WT, Lachance MA (2011) Yeast Ecology. In: Kurtzman CP, Fell JW, Boekhout T (eds) The Yeasts, 5th 1124 edn. Elsevier, San Diego, CA, pp 65-83

1125 Steinhaus E (1941) A study of the bacteria associated with thirty species of insects. J Bacteriol 42:757-90. 
1126 Stoll S, Gadau J, Gross R, Feldhaar H (2007) Bacterial microbiota associated with ants of the genus Tetraponera.

1127 Biol J Linn Soc 90:399-412. doi: 10.1111/j.1095-8312.2006.00730.x

1128 Suh S-O, McHugh J V, Pollock DD, Blackwell M (2005) The beetle gut: a hyperdiverse source of novel yeasts.

$1129 \quad$ Mycol Res 109:261-265. doi: 10.1017/S0953756205002388

1130 Sullam KE, Essinger SD, Lozupone CA, et al (2012) Environmental and ecological factors that shape the gut

1131 bacterial communities of fish: a meta-analysis. Mol Ecol 21:3363-3378. doi: 10.1111/j.1365-

$1132 \quad$ 294X.2012.05552.x

1133 Tap J, Mondot S, Levenez F, et al (2009) Towards the human intestinal microbiota phylogenetic core. Environ

$1134 \quad$ Microbiol 11:2574-2584. doi: 10.1111/j.1462-2920.2009.01982.x

1135 Wang Q, Garrity GM, Tiedje JM, Cole JR (2007) Naïve Bayesian classifier for rapid assignment of rRNA sequences

1136 into the new bacterial taxonomy. Appl Environ Microbiol 73:5261-5267. doi: 10.1128/AEM.00062-07

1137 Warnecke F, Luginbühl P, Ivanova N, et al (2007) Metagenomic and functional analysis of hindgut microbiota of a

1138 wood-feeding higher termite. Nature 450:560-565. doi: 10.1038/nature06269

1139 White TJ, Bruns T, Lee S, Taylor J (1990) Amplification and direct sequencing of fungal ribosomal RNA genes for

1140 phylogenetics. PCR Protoc A Guid to Methods Appl. doi: http://dx.doi.org/10.1016/B978-0-12-372180-

$11418.50042-1$

1142 Xiang H, Wei G, Jia S (2006) Microbial communities in the larval midgut of laboratory and field populations of

1143 cotton bollworm (Helicoverpa armigera). Can J Microbiol 52:1085-1092. doi: 10.1139/W06-064

1144 Zhang Y, Schoch CL, Fournier J, et al (2009) Multi-locus phylogeny of Pleosporales: A taxonomic, ecological and

1145 evolutionary re-evaluation. Stud Mycol 64:85-102. doi: 10.3114/sim.2009.64.04

1146 Zuur AF, Ieno EN, Walker NJ, et al (2009) Mixed Effects Models and Extensions in Ecology with R. Springer, New $1147 \quad$ York

1148 
1149 Table 1. Sample sizes

1150

1151 Number of butterfly or food samples ("individuals") and species remaining in the sequencing 1152 datasets after rarefying.

1153

1154

\begin{tabular}{l|c|c|c|c|}
\cline { 2 - 5 } & \multicolumn{2}{c|}{ Bacterial dataset } & \multicolumn{2}{c|}{ Fungal dataset } \\
\cline { 2 - 5 } & Individuals & Species & Individuals & Species \\
\cline { 2 - 5 } Frugivores & 148 & 24 & 92 & 15 \\
\cline { 2 - 5 } Nectivores & 142 & 28 & 69 & 19 \\
\cline { 2 - 5 } Fruits & 12 & 1 & 13 & 1 \\
\cline { 2 - 5 } Nectars & 46 & 8 & 39 & $\mathrm{n} / \mathrm{a}$ \\
\cline { 2 - 5 } Trap baits & 25 & $\mathrm{n} / \mathrm{a}$ & 14 &
\end{tabular}


1156

1157

1158

1159

1160

1161

1162

Table 2. Taxonomic identities of the 20 most abundant bacterial OTUs

The 20 most abundant bacterial OTUs. "\% Total" is the percentage of sequences (pooled across all butterflies) that were assigned to the OTU. "\% Butterflies", "\% Frugivores", and "\% Nectivores" are the percentages of all butterflies, frugivores, and nectivores in which the OTU was detected. Taxonomy was assigned by the RDP classifier using the Greengenes training set, except as otherwise noted. Confidences measure the degree of certainty of the taxonomic assignment.

\begin{tabular}{|c|c|c|c|c|c|c|c|c|c|c|c|c|c|}
\hline OTU ID & \% Reads & \% Butterflies & \% Frugivores & $\%$ Nectivores & Frug:Nect $F$ & Phylum & Class & Order & Family & Genus & Species & Confidence & Notes \\
\hline OTU_1 & 9.8 & 73 & 84 & 63 & 1.3 & Proteobacteria & Gammaproteobacteria & Orbales & Orbaceae & Orbus & & 100 & a \\
\hline OTU_4 & 8.7 & 82 & 81 & 84 & 1.0 & Proteobacteria & Gammaproteobacteria & Enterobacteriales & Enterobacteriaceae & Klebsiella/Enterobacter & & 100 & b \\
\hline OTU_3 & 8.3 & 56 & 42 & 70 & 0.6 & Proteobacteria & Alphaproteobacteria & Rhodospirillales & Acetobacteraceae & Swaminathania/Asaia & & 97 & c \\
\hline OTU_6 & 6.0 & 83 & 86 & 80 & 1.1 & Firmicutes & Bacilli & Lactobacillales & Enterococcaceae & Vagococcus/Enterococcus & & 62 & d \\
\hline OTU_2 & 4.8 & 44 & 41 & 48 & 0.9 & Bacteroidetes & Flavobacteriia & Flavobacteriales & [Weeksellaceae] & & & 96 & \\
\hline OTU_19 & 3.2 & 46 & 34 & 59 & 0.6 & Proteobacteria & Gammaproteobacteria & Enterobacteriales & Enterobacteriaceae & Serratia & marcescens & 94 & \\
\hline OTU_5 & 2.7 & 28 & 27 & 29 & 0.9 & Tenericutes & Mollicutes & Entomoplasmatales & & Spiroplasma/Entomoplasma & & 100 & e \\
\hline OTU_10 & 2.6 & 28 & 36 & 20 & 1.8 & Bacteroidetes & Bacteroidia & Bacteroidales & Porphyromonadaceae & & & 68 & \\
\hline OTU_9 & 2.5 & 30 & 27 & 34 & 0.8 & Proteobacteria & Alphaproteobacteria & Rhizobiales & Bartonellaceae & Bartonella & & 92 & $f$ \\
\hline OTU_12 & 2.5 & 44 & 40 & 49 & 0.8 & Firmicutes & Bacilli & Lactobacillales & Streptococcaceae & Lactococcus & & 100 & \\
\hline OTU_18 & 2.3 & 46 & 72 & 19 & 3.8 & Proteobacteria & Alphaproteobacteria & Rhodospirillales & Acetobacteraceae & Acetobacter & & 100 & $\mathrm{~g}$ \\
\hline OTU_11 & 2.2 & 34 & 46 & 22 & 2.1 & Proteobacteria & Gammaproteobacteria & Orbales & Orbaceae & Gilliamella/Orbus & & 91 & $\mathrm{~h}$ \\
\hline OTU_17 & 2.2 & 27 & 36 & 18 & 2.0 & Proteobacteria & Alphaproteobacteria & Rhodospirillales & Acetobacteraceae & Commensalibacter & intestini & 95 & \\
\hline OTU_14 & 1.7 & 19 & 25 & 13 & 1.9 & Proteobacteria & Gammaproteobacteria & Enterobacteriales & Enterobacteriaceae & Providencia/Serratia & & 73 & $\mathrm{i}$ \\
\hline OTU_13 & 1.7 & 18 & 22 & 13 & 1.7 & Proteobacteria & Alphaproteobacteria & Rickettsiales & Rickettsiaceae & Wolbachia & & 100 & \\
\hline OTU_15 & 1.6 & 26 & 31 & 20 & 1.5 & Proteobacteria & Gammaproteobacteria & Pseudomonadales & Pseudomonadaceae & & & 73 & \\
\hline OTU_23 & 1.4 & 23 & 26 & 19 & 1.4 & Proteobacteria & Gammaproteobacteria & Pasteurellales & & & & 100 & \\
\hline OTU_569 & 1.4 & 27 & 18 & 37 & 0.5 & Proteobacteria & Gammaproteobacteria & Enterobacteriales & Enterobacteriaceae & Erwinia/Pantoea & & 56 & $\mathrm{j}$ \\
\hline OTU_22 & 1.4 & 25 & 23 & 27 & 0.9 & Proteobacteria & Alphaproteobacteria & Rhodospirillales & Acetobacteraceae & Commensalibacter & intestini & 93 & \\
\hline OTU_1106 & 1.2 & 19 & 16 & 21 & 0.8 & Bacteroidetes & Flavobacteriia & Flavobacteriales & [Weeksellaceae] & & & 68 & \\
\hline
\end{tabular}

Notes

a. RDP assigned to the order Pasteurellales with no finer resolution. Closest NCBI hit was Orbales:Orbaceae:Orbus.

b. In a blast search against NCBI, matched equally closely to the genera Klebsiella and Enterobacter.

c. RDP assigned to genus Swaminanthia; closest NCBI hit was Asaia.

d. Closest NCBI hit was Enterococcus

e. In the blast search against NCBI, matched equally closely to the genera Spiroplasma and Entomoplasma

f. RDP assigned to family with no finer resolution. Closest NCBI match was the genus Bartonella.

g. Closest NCBI species matches were indonesiensis or okinawensis.

h. RDP assigned to the order Pasteurellales with no finer resolution. Closest NCBI hit was Orbales:Orbaceae:Gilliamella/Orbus.

i. RDP assigned to family with no finer resolution. Closest NCBI matches were the genera Providencia and Serratia.

1163

j. Closest NCBI match was the genus Pantoea. 
Table 3. Taxonomic identities of the 20 most abundant fungal OTUs

The 20 abundant fungal OTUs detected in at least $10 \%$ of the butterfly samples. " $\%$ Total" is the percentage of sequences (pooled across all butterflies) that were assigned to the OTU. "\% Butterflies", "\% Frugivores", and "\% Nectivores" are the percentages of all butterflies, frugivores, and nectivores in which the OTU was detected. Taxonomy was assigned by the RDP classifier using the Warcup training set. Where a rank is incertae sedis, the closest available rank is written in brackets. Confidences measure the degree of certainty of the taxonomic assignment and are cumulative from higher to lower taxonomic ranks. Assignments with particularly low confidence are greyed out. (One additional fungal OTU, a Torulaspora species, was observed at high abundance in the pooled data but was detected in less than $10 \%$ of frugivores and less than $10 \%$ of nectivores, so is omitted here.)

\begin{tabular}{|c|c|c|c|c|c|c|c|c|c|c|c|c|c|c|c|c|c|}
\hline OTU ID & \% Reads & \% Butterflies & \% Frugivores & \% Nectivores & Frug:Nect & Phylum & Pconf & f Class & Cconf & Order & Oconf & Family & Fconf & Genus & Gconf & Species & Sconf \\
\hline OTU_7 & 5.0 & 22 & 7 & 37 & 0.2 & Zygomycota & 20 & [Mucoromycotina] & 15 & Mucorales & 15 & Mucoraceae & 7 & Rhizopus & 6 & caespitosus & 6 \\
\hline OTU_10 & 3.7 & 13 & 9 & 16 & 0.6 & Basidiomycota & 68 & Agaricostilbomycetes & 29 & Agaricostilbales & 29 & Agaricostilbaceae & 27 & Bensingtonia & 27 & yuccicola & 9 \\
\hline DTU_20 & 3.2 & 9 & 12 & 3 & 4.0 & Ascomycota & 68 & Saccharomycetes & 13 & Saccharomycetales & 13 & Saccharomycodaceae & 3 & Hanseniaspora & 3 & uvarum & 3 \\
\hline OTU_17 & 2.9 & 45 & 56 & 25 & 2.2 & Ascomycota & 100 & Saccharomycetes & 100 & Saccharomycetales & 100 & Saccharomycodaceae & 100 & Hanseniaspora & 100 & uvarum & 94 \\
\hline DTU_2469 & 2.6 & 42 & 57 & 16 & 3.6 & Ascomycota & 100 & Saccharomycetes & 100 & Saccharomycetales & 100 & Saccharomycodaceae & 100 & Hanseniaspora & 100 & opuntiae & 62 \\
\hline OTU_25 & 2.4 & 35 & 51 & 11 & 4.6 & Ascomycota & 100 & Saccharomycetes & 100 & Saccharomycetales & 100 & Pichiaceae & 99 & Pichia & 99 & fermentans & 70 \\
\hline OTU_2439 & 2.2 & 21 & 28 & 9 & 3.1 & Ascomycota & 100 & Saccharomycetes & 100 & Saccharomycetales & 100 & Saccharomycetaceae & 100 & Kazachstania & 100 & exigua & 100 \\
\hline OTU_11 & 1.9 & 29 & 34 & 18 & 1.9 & Ascomycota & 100 & Saccharomycetes & 100 & Saccharomycetales & 100 & Saccharomycetales_Incertae sedis & 97 & Candida & 97 & quercitrusa & 96 \\
\hline OTU_2306 & 1.9 & 40 & 53 & 18 & 2.9 & Ascomycota & 100 & Saccharomycetes & 100 & Saccharomycetales & 100 & Saccharomycodaceae & 100 & Hanseniaspora & 100 & guilliermondii & 42 \\
\hline OTU_2503 & 1.5 & 15 & 6 & 24 & 0.2 & Ascomycota & 35 & Saccharomycetes & 7 & Saccharomycetales & 7 & Saccharomycodaceae & 5 & Hanseniaspora & 5 & uvarum & 5 \\
\hline OTU_78 & 1.5 & 21 & 16 & 25 & 0.6 & Ascomycota & 97 & Leotiomycetes & 90 & [Leotiomycetes] & 32 & [Leotiomycetes] & 32 & Leohumicola & 17 & levissima & 14 \\
\hline OTU_24 & 1.5 & 13 & 15 & 9 & 1.7 & Ascomycota & 99 & Saccharomycetes & 98 & Saccharomycetales & 98 & Saccharomycetaceae & 98 & Kazachstania & 96 & hellenica & 96 \\
\hline OTU_41 & 1.4 & 8 & 3 & 13 & 0.2 & Basidiomycota & 46 & Microbotryomycetes & 8 & Sporidiobolales & 7 & [Sporidiobolales] & 7 & Rhodotorula & 1 & cresolica & 0 \\
\hline OTU_127 & 1.2 & 14 & 7 & 20 & 0.4 & Ascomycota & 94 & Dothideomycetes & 80 & Pleosporales & 75 & [Pleosporales] & 38 & Clavariopsis & 37 & aquatica & 37 \\
\hline OTU_997 & 1.2 & 13 & 22 & 0 & $n / a$ & Ascomycota & 100 & Saccharomycetes & 100 & Saccharomycetales & 100 & Saccharomycetaceae & 100 & Kazachstania & 100 & exigua & 100 \\
\hline OTU_151 & 1.2 & 5 & 0 & 11 & 0 & Ascomycota & 43 & Saccharomycetes & 10 & Saccharomycetales & 10 & Saccharomycetaceae & 5 & Kazachstania & 2 & hellenica & 2 \\
\hline OTU_1735 & 1.1 & 9 & 10 & 7 & 1.4 & Ascomycota & 78 & Saccharomycetes & 40 & Saccharomycetales & 40 & Pichiaceae & 17 & Pichia & 10 & haplophila & 7 \\
\hline OTU_3 & 1.1 & 13 & 6 & 20 & 0.3 & Ascomycota & 22 & Saccharomycetes & 18 & Saccharomycetales & 18 & [Saccharomycetales] & 11 & Cyberlindnera & 5 & fabianii & 5 \\
\hline OTU_96 & 1.0 & 11 & 6 & 14 & 0.4 & Ascomycota & 100 & Saccharomycetes & 100 & Saccharomycetales & 100 & Pichiaceae & 100 & Pichia & 100 & jadinii & 100 \\
\hline OTU_73 & 1.0 & 16 & 11 & 20 & 0.5 & Ascomycota & 100 & [Pezizomycotina] & 19 & [Pezizomycotina] & 19 & [Pezizomycotina] & 19 & Fontanospora & 17 & fusiramosa & 17 \\
\hline
\end{tabular}


Figure 1. Total bacterial load per butterfly by species.

Total bacterial 16s counts per butterfly were estimated via qPCR. Host species are arranged in ascending size (wing length) along the $\mathrm{x}$-axis. Dots indicate copy number for individual frugivores (red) or nectivores (blue), and boxplots depict medians and interquartile ranges of the data. Whiskers are placed at 1.5 times the interquartile range or, if all data fall within this range, they are placed at most extreme value measured. Note that although feeding guild is displayed, it was not a significant predictor of total bacterial load.

Total bacterial load per butterfly by species

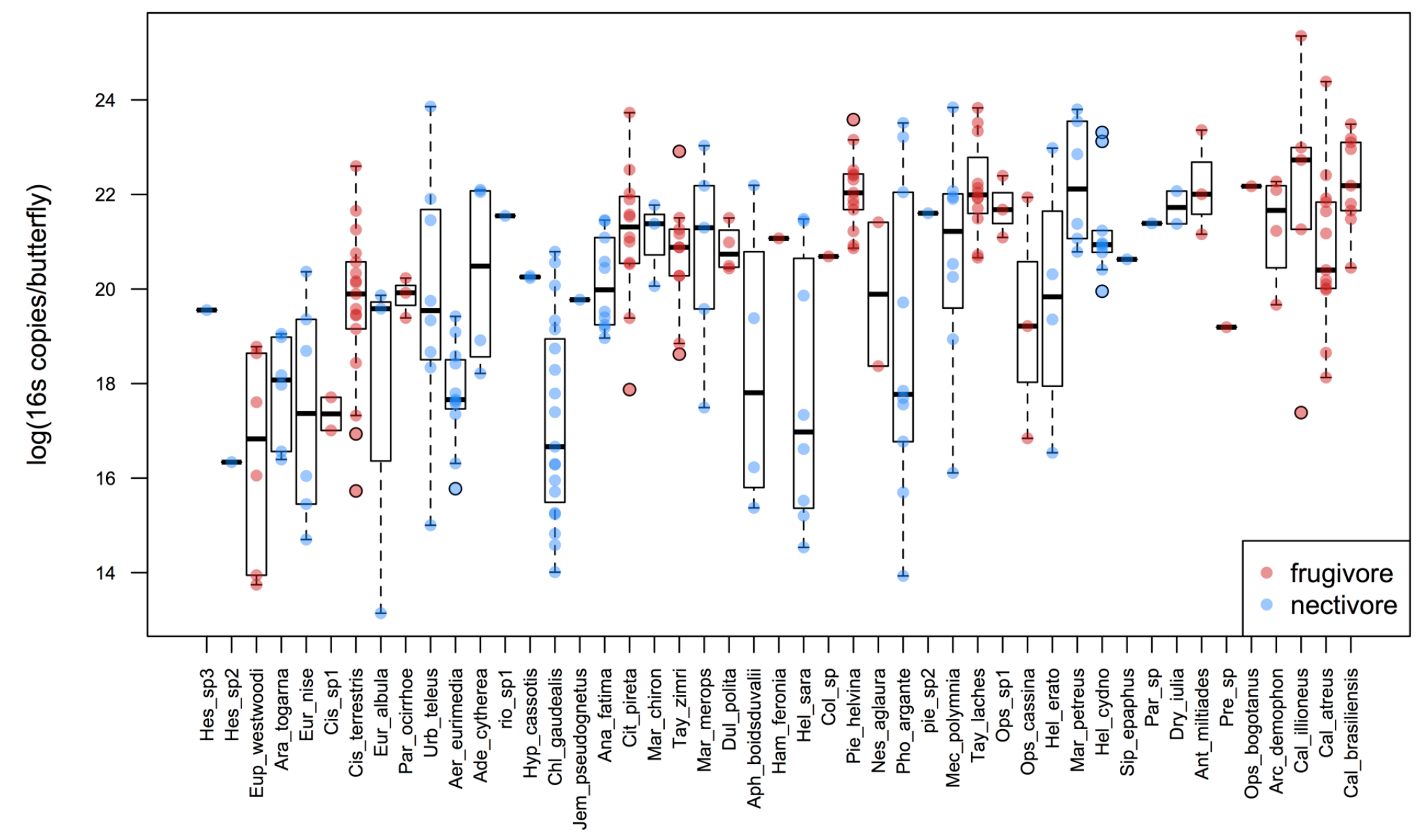


Figure 2. Microbial species richness in frugivores and nectivores.

Bacterial (a) and fungal (b) observed species richness per butterfly did not differ between frugivores and nectivores. P-values are the result of model comparisons and control for host species (see text). Boxplot features are as described in Figure 1. One outlier of 162 bacterial OTUs in a nectivore is not shown in panel (a). Note that since bacteria and fungi were rarefied to different cutoffs, species richness estimates are not comparable between the two.

a)

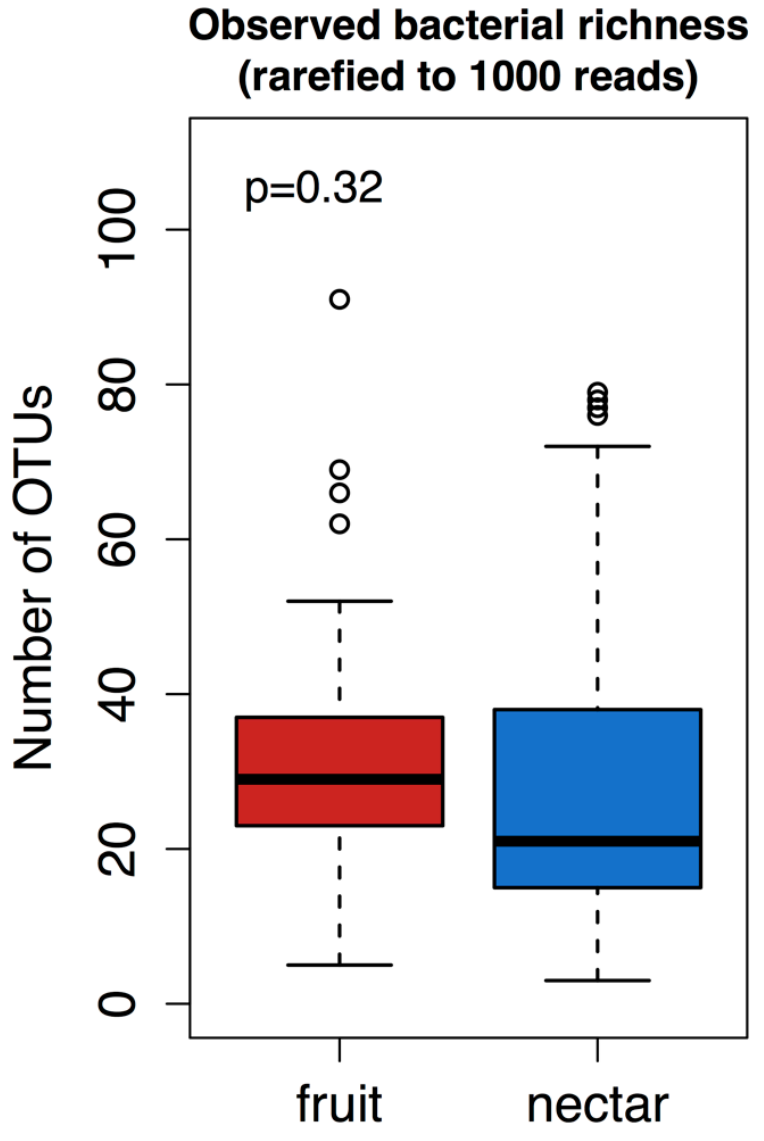

b)

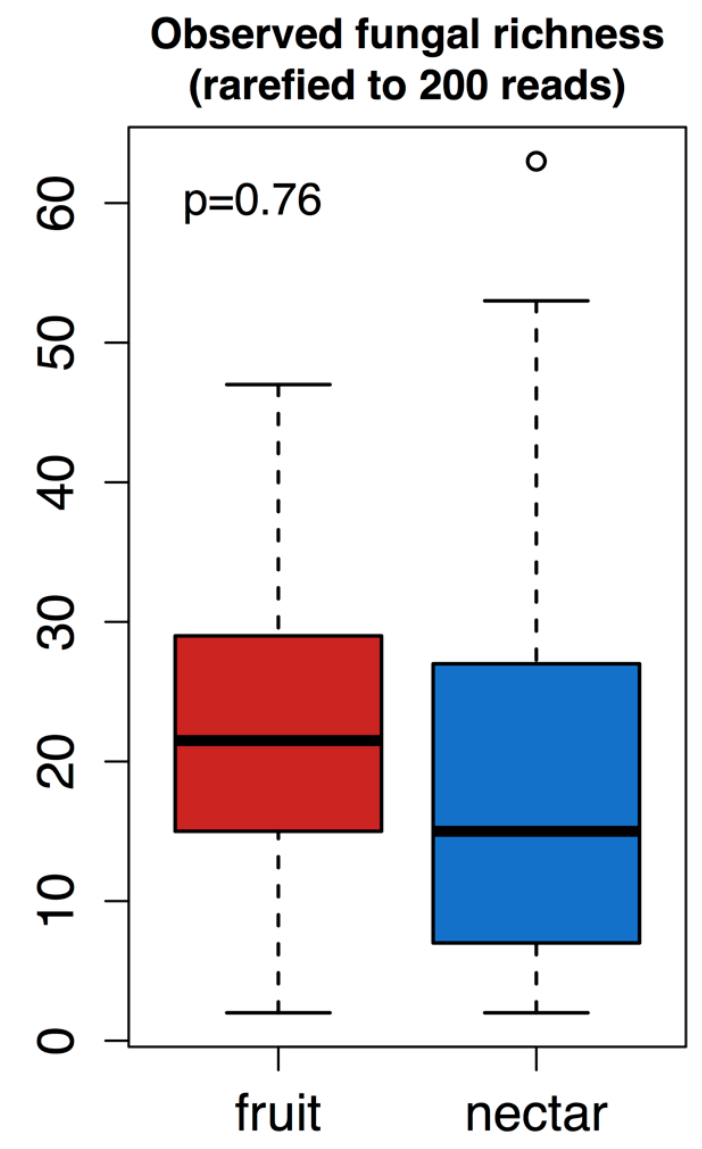


Figure 3. Ordinations of bacterial and fungal community composition.

NMDS plots of the Bray-Curtis dissimilarities between butterflies. PerMANOVA tests confirmed that the gut flora of frugivores and nectivores differed in bacterial and fungal OTU composition (p-values displayed on plots). (a) Bacterial gut flora of 148 frugivores and 142 nectivores. (b) Fungal gut flora of 92 frugivores and 69 nectivores. One outlier is not displayed in panel (b).

(a)

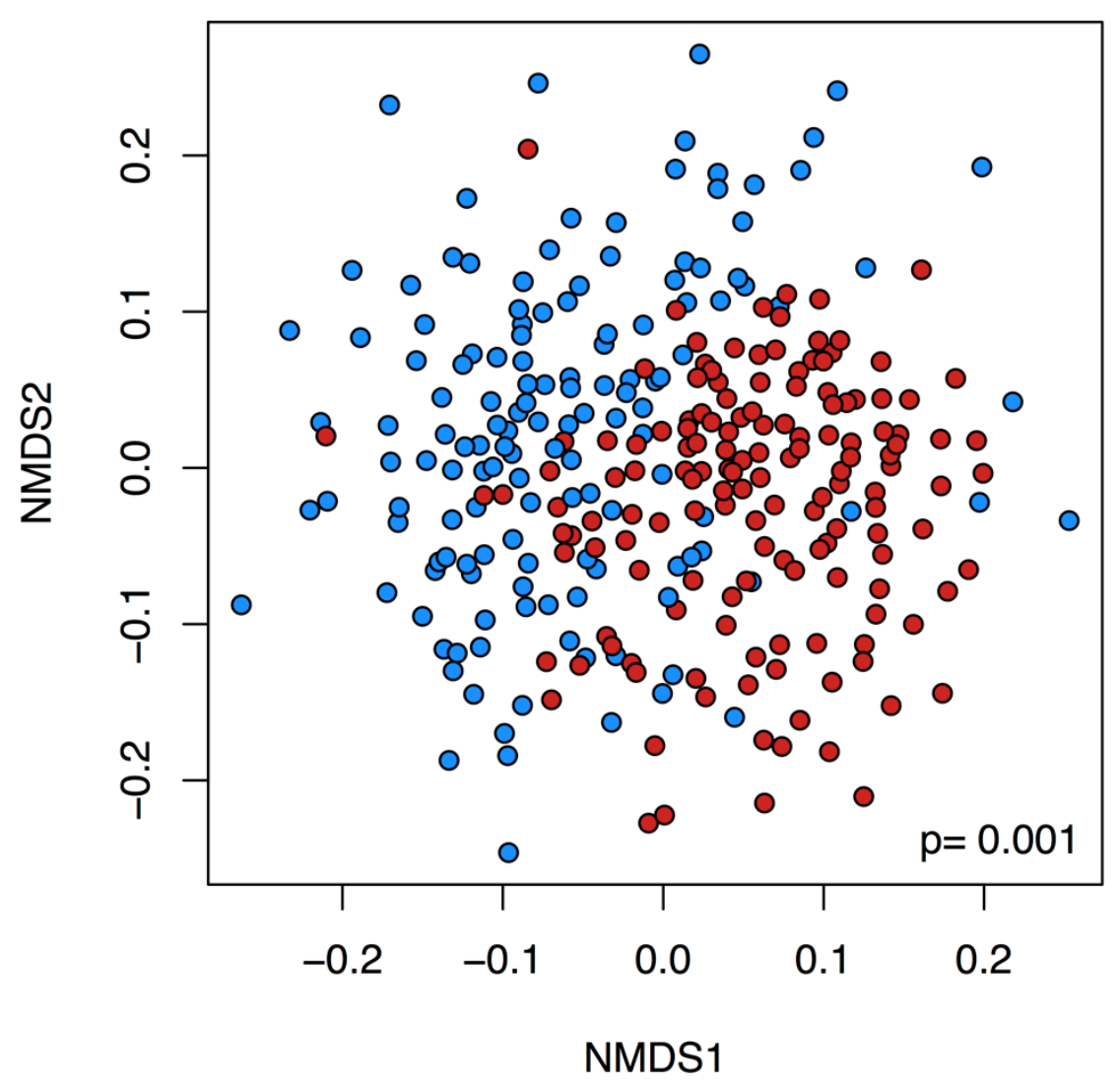

(b)

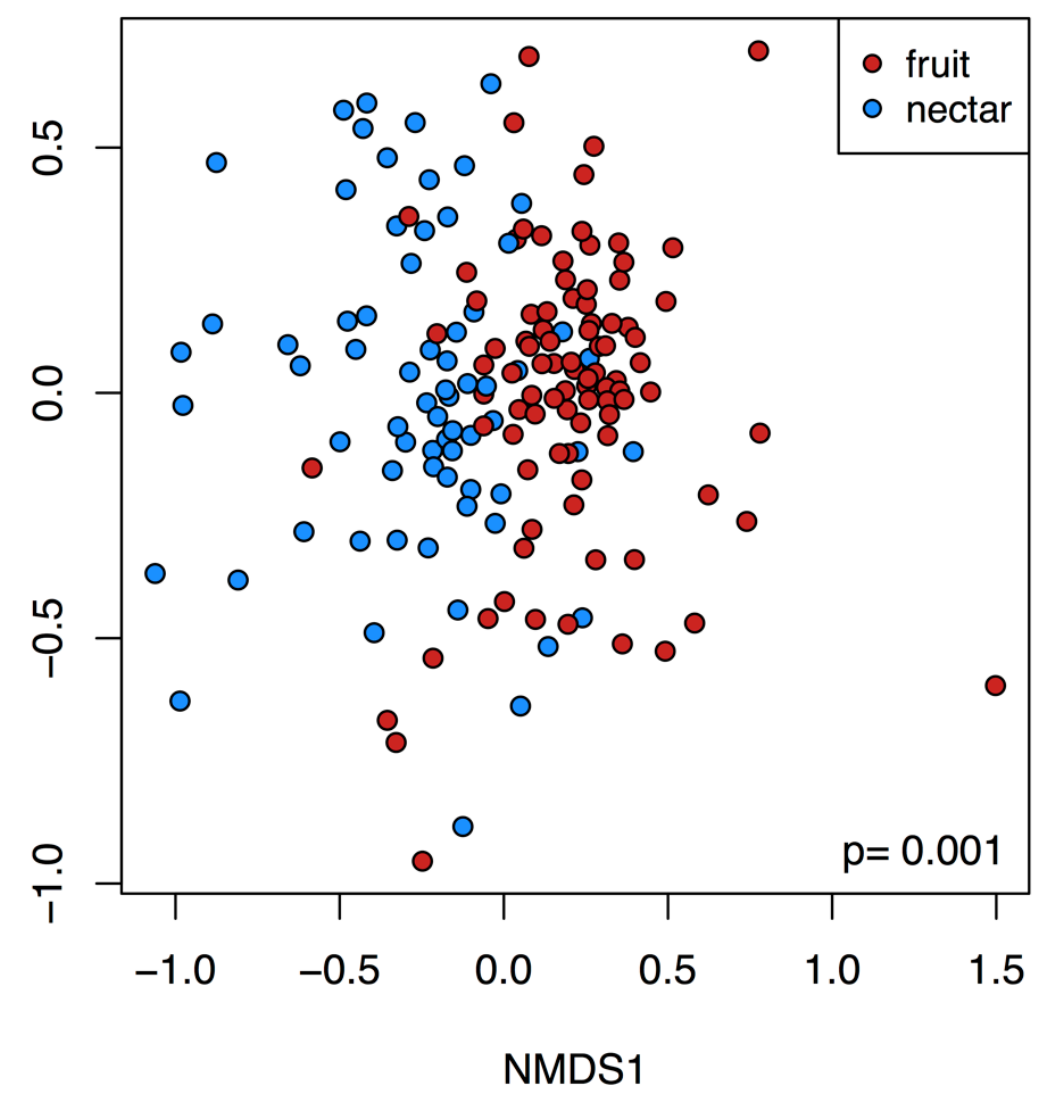


Figure 4. Effect of host diet (a) and host species (b) on bacterial community composition.

(a) Model-predicted estimates of the mean relative abundance per butterfly (counts out of 1000) of the 20 most abundant bacterial OTUs in the dataset. Relative abundance was modeled as a function of the interaction between host diet and OTU identity, with a random intercept of host species. Points indicate the model estimated mean and lines indicate one standard error. OTUs are arranged along the $\mathrm{x}$-axis according to the magnitude and direction of the difference in their abundance between frugivores and nectivores. Each OTU is designated by genus or by the finest taxonomic resolution available, followed by OTU number in parentheses. The shaded gray box indicates OTUs that did not differ in abundance between the feeding guilds.

(b) Mean relative abundances of the 20 most abundant bacterial OTUs per host species. Darker shading indicates higher mean relative abundance.

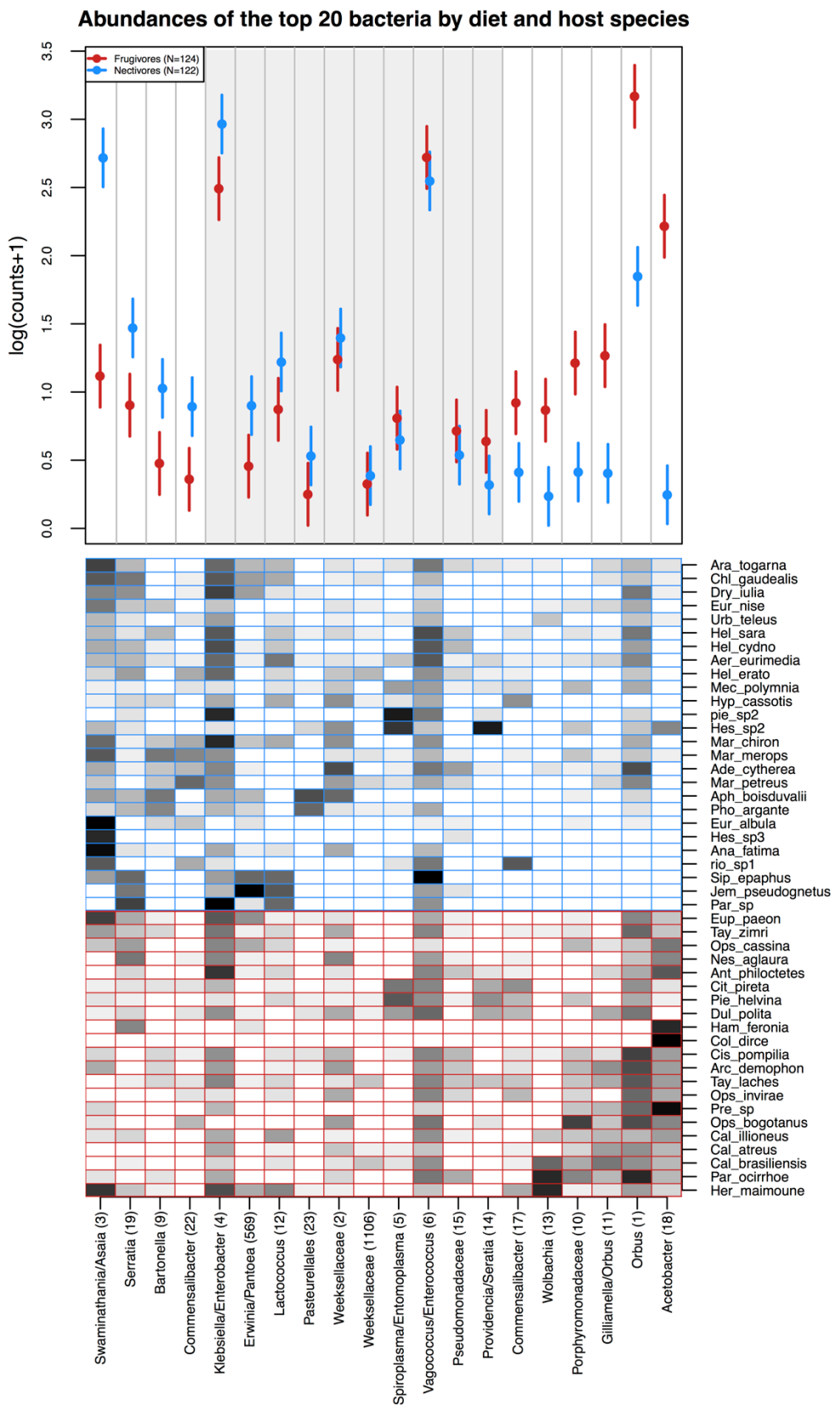


Figure 5. Effect of host diet (a) and host species (b) on fungal community composition.

(a) Model-predicted estimates of the mean relative abundance per butterfly (counts out of 1000) of the 20 most abundant fungal OTUs in the dataset. Relative abundance was modeled as a function of the interaction between host diet and OTU identity, with a random effect of host species. Points indicate the predicted mean and lines indicate one standard error. OTUs are arranged along the $\mathrm{x}$-axis according to the magnitude and direction of the difference in their abundance between frugivores and nectivores. Each OTU is designated by genus or by the finest taxonomic resolution available, followed by OTU number in parentheses. The shaded gray box indicates OTUs that did not differ in abundance between the feeding guilds.

(b) Mean relative abundances of the 20 most abundant fungal OTUs per host species. Darker shading indicates higher mean relative abundance.

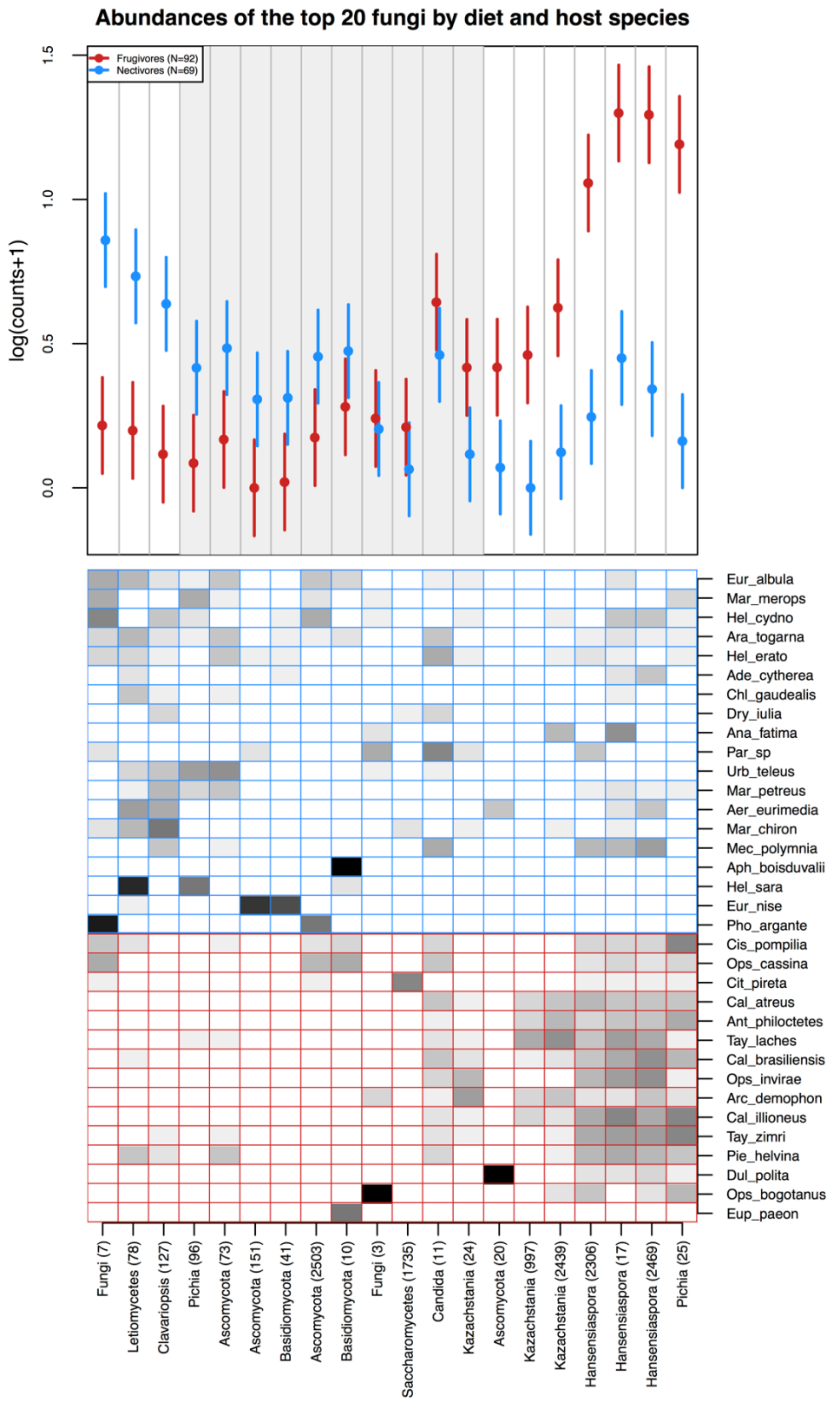


Figure 6. Dissimilarity of gut flora within versus between host species.

Every point represents the average Bray-Curtis dissimilarity between of a given individual's gut community and the gut communities of all other conspecific hosts (green) or all heterospecific hosts (orange). Boxplot features are as described in Figure 1. P-values are the results of t-tests for pairwise differences in dissimilarity.

\section{Dissimilarity of gut flora within versus between host species}

a) Bacteria

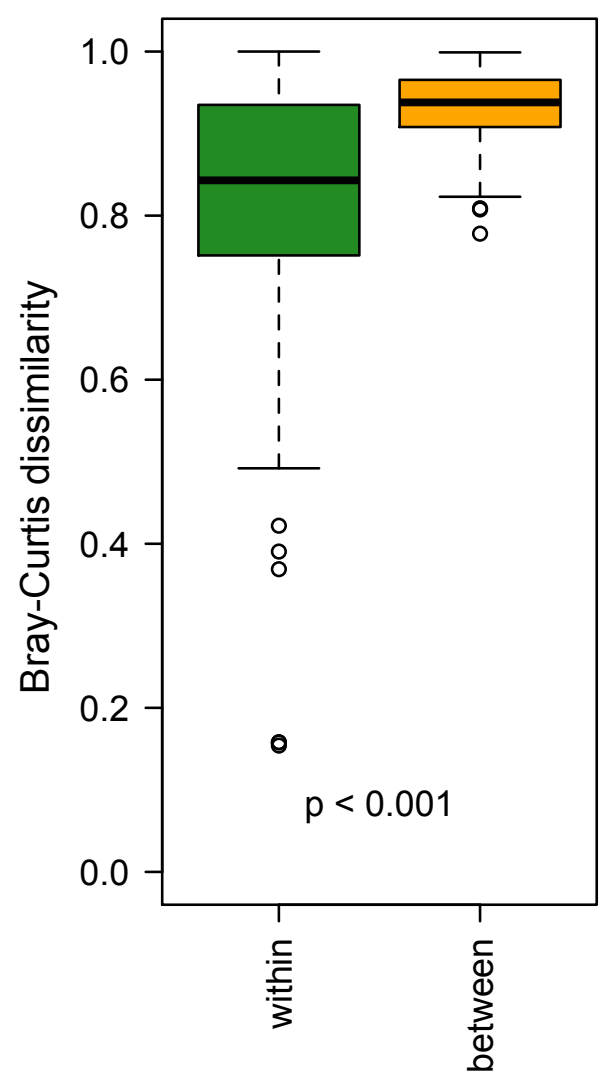

b) Fungi

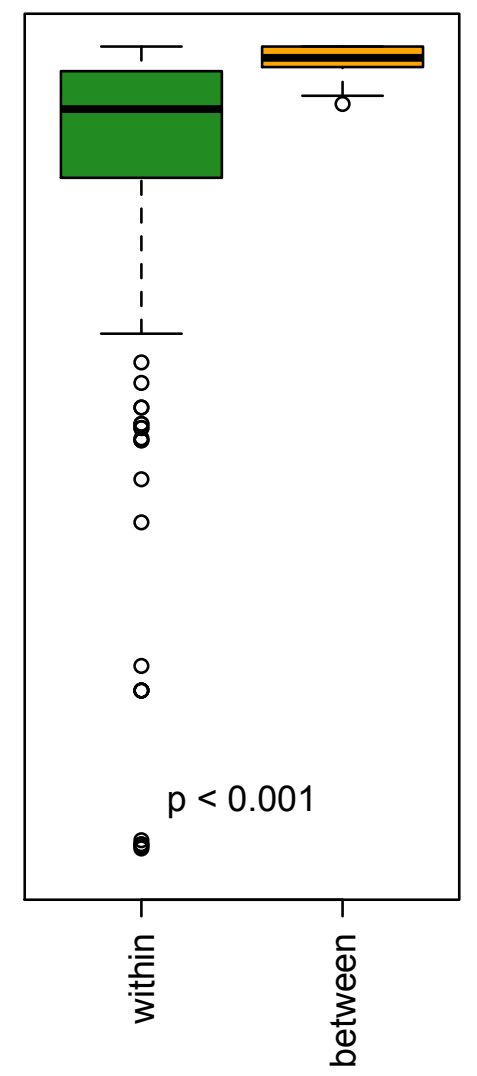


Figure 7. Comparison of frugivore and nectivore gut communities to the microbial communities of butterfly foods.

NMDS plots of the Bray-Curtis dissimilarities between butterflies and their foods. (a) Bacterial flora of 149 frugivores, 149 nectivores, 46 nectars, 25 trap baits, and 12 fruits. (b) Fungal gut flora of 94 frugivores, 70 nectivores, 39 nectars, 14 trap baits, and 13 fruits. Two samples were fungal outliers and are not displayed in panel (b). (Sample sizes differ slightly from Figure 3 because in order to include as many food samples as possible, the combined gut and food sequence data were rarefied to different depths than the gut sequence data alone.)

(a)

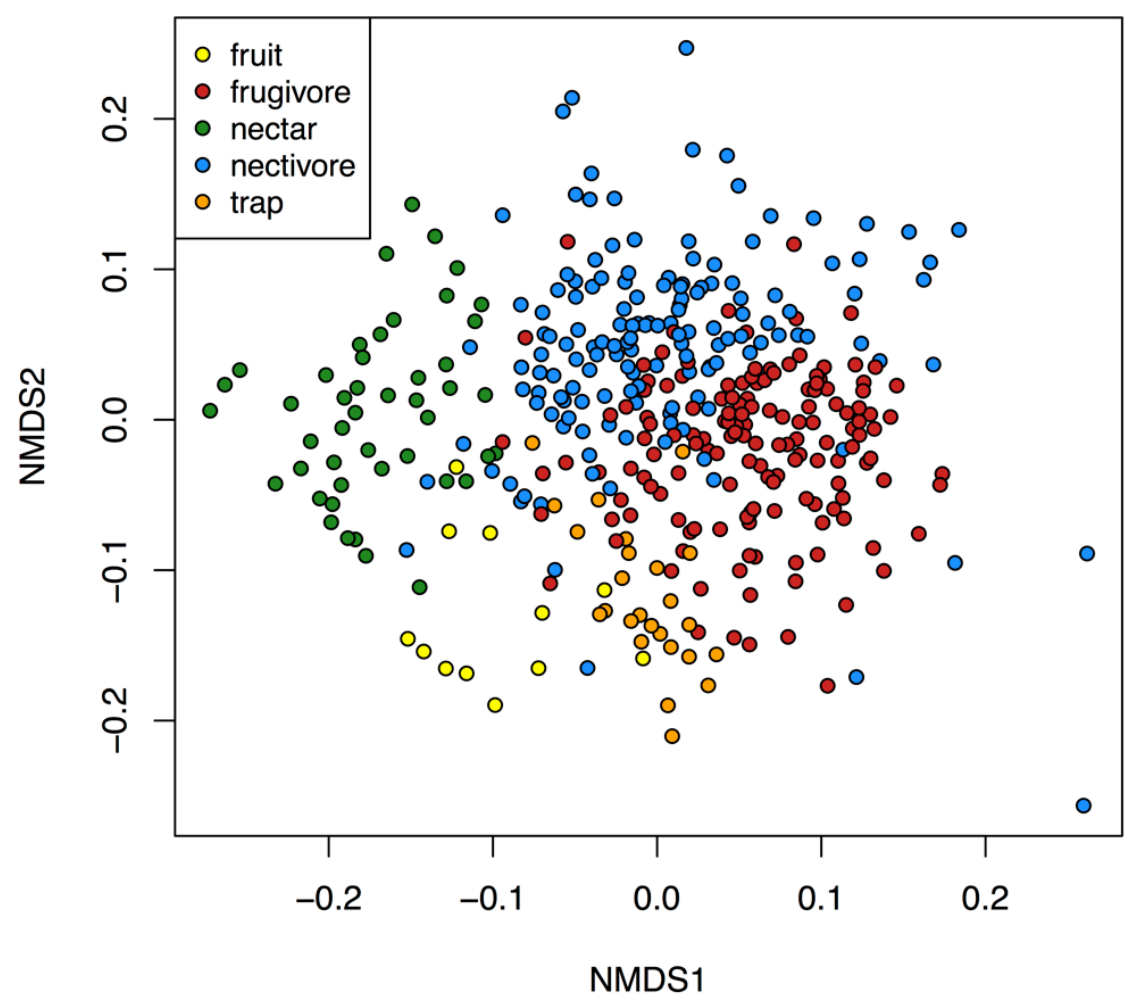

(b)

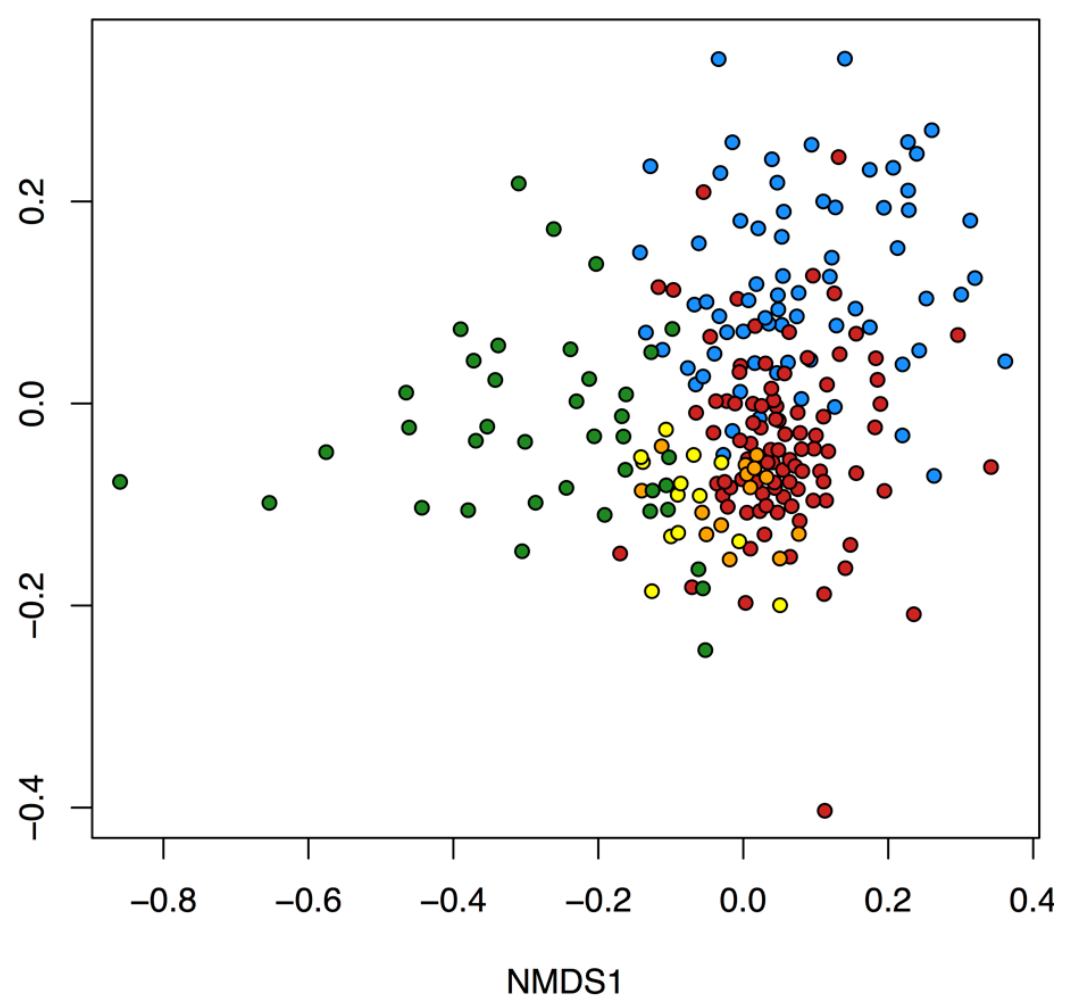


Figure 8. Dissimilarity between gut and food microbial communities.

Bray-Curtis dissimilarities between butterfly gut bacterial (a) and fungal (b) communities and the microbial communities in butterfly foods. Every point represents the average distance of a butterfly gut community to all food communities in a given category (fruit, trap bait, or nectar). Comparisons to frugivorous butterflies are colored red; comparisons to nectivores are colored blue. Boxplot features are as described in Figure 1. P-values are the results of t-tests for pairwise differences in dissimilarity; these have been FDR-corrected.

Note that three fungal strains of Kazachstania exigua were excluded from the butterfly data but not the food data, since these OTUs were introduced into frugivores' guts via the trap baits. (Described in the main text.) Frugivores would appear more similar to the trap baits if these OTUs had been included.

Dissimilarity between gut and food microbial communities

a) Bacteria

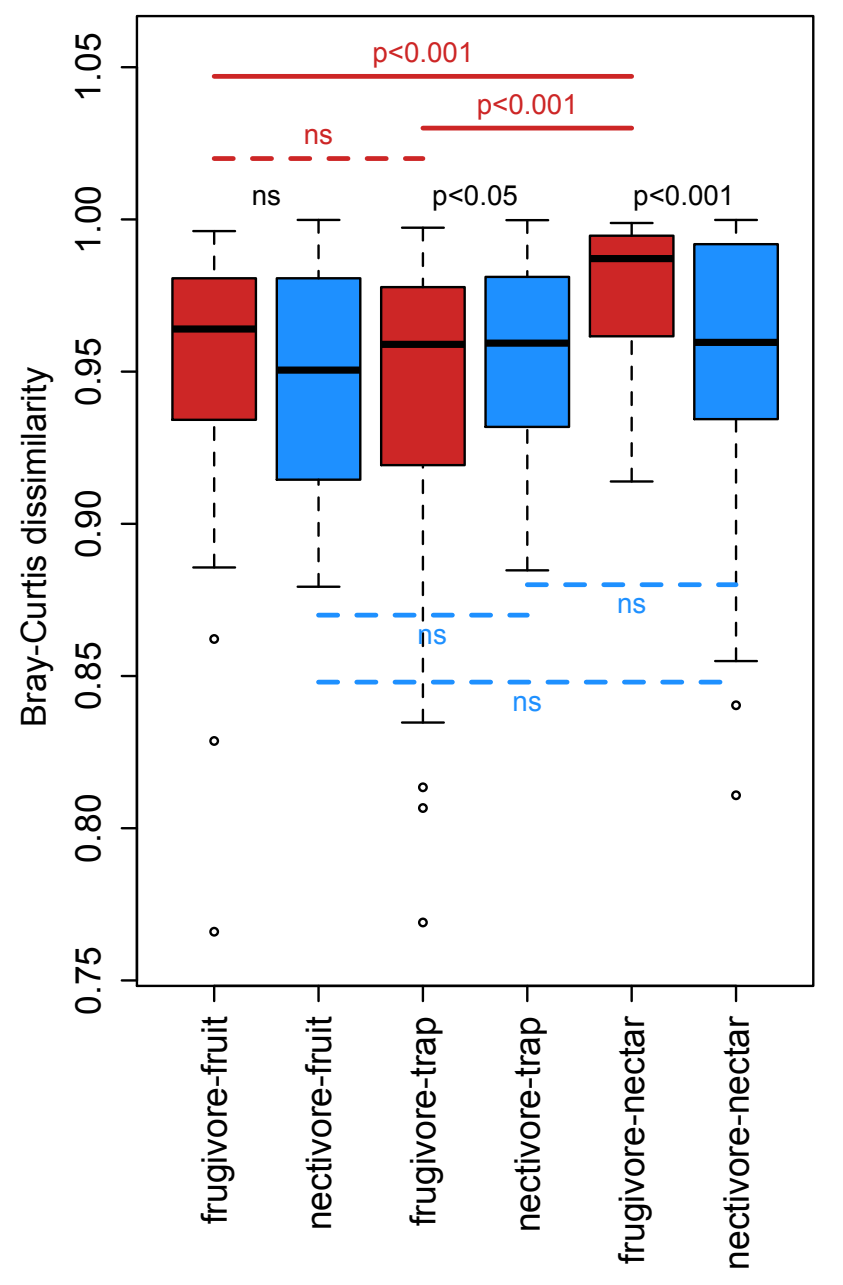

b) Fungi

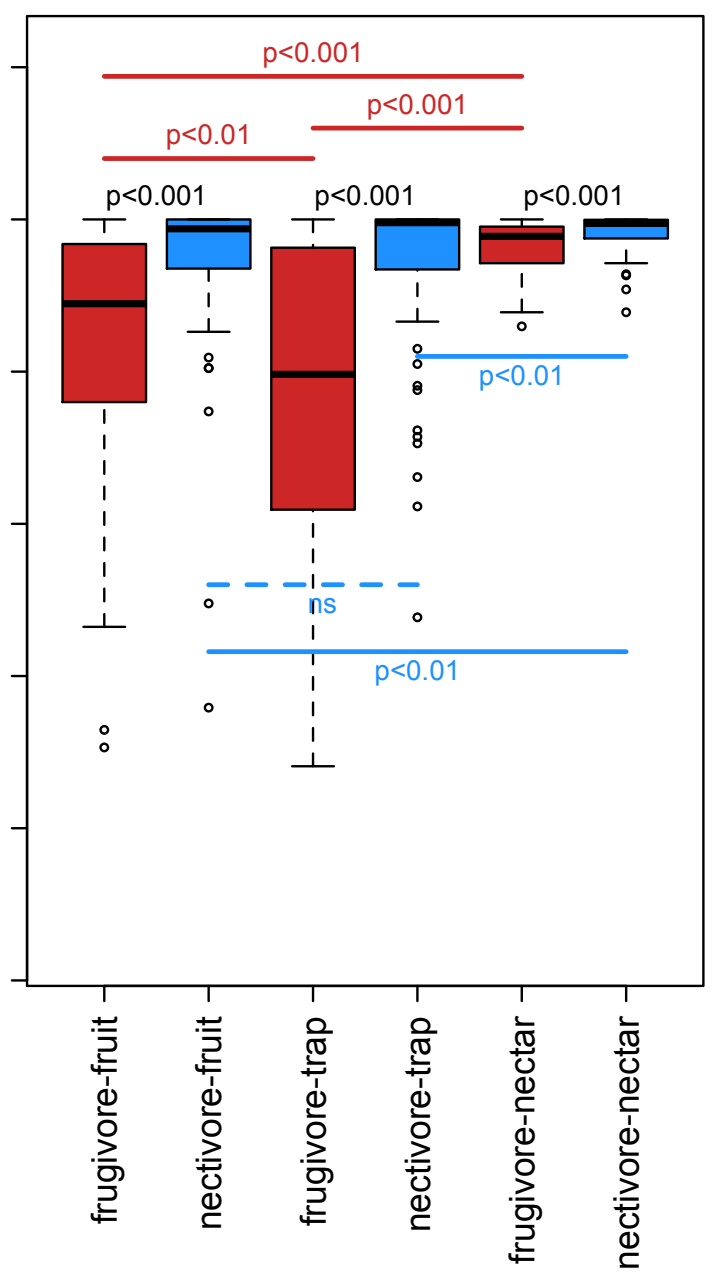


Figure 9. Differences in microbial community catabolism between frugivores and nectivores.

Points indicate the model-estimated mean difference in catabolism of a substrate (units of absorbance at $590 \mathrm{~nm}$, standardized by plate and Hellinger transformed) between frugivores' and nectivores' gut microbial communities. Gut community catabolism of substrates in dark red and dark blue was significantly greater in frugivores or nectivores, respectively, after multiple test correction. Light red and light blue substrates were significantly different between guilds before, but not after multiple test correction. Thick lines and points indicate model-estimated means and standard errors for substrate classes. Red indicates classes that were catabolized more strongly by frugivore gut communities; blue indicates classes that were digested more actively by nectivore gut flora.

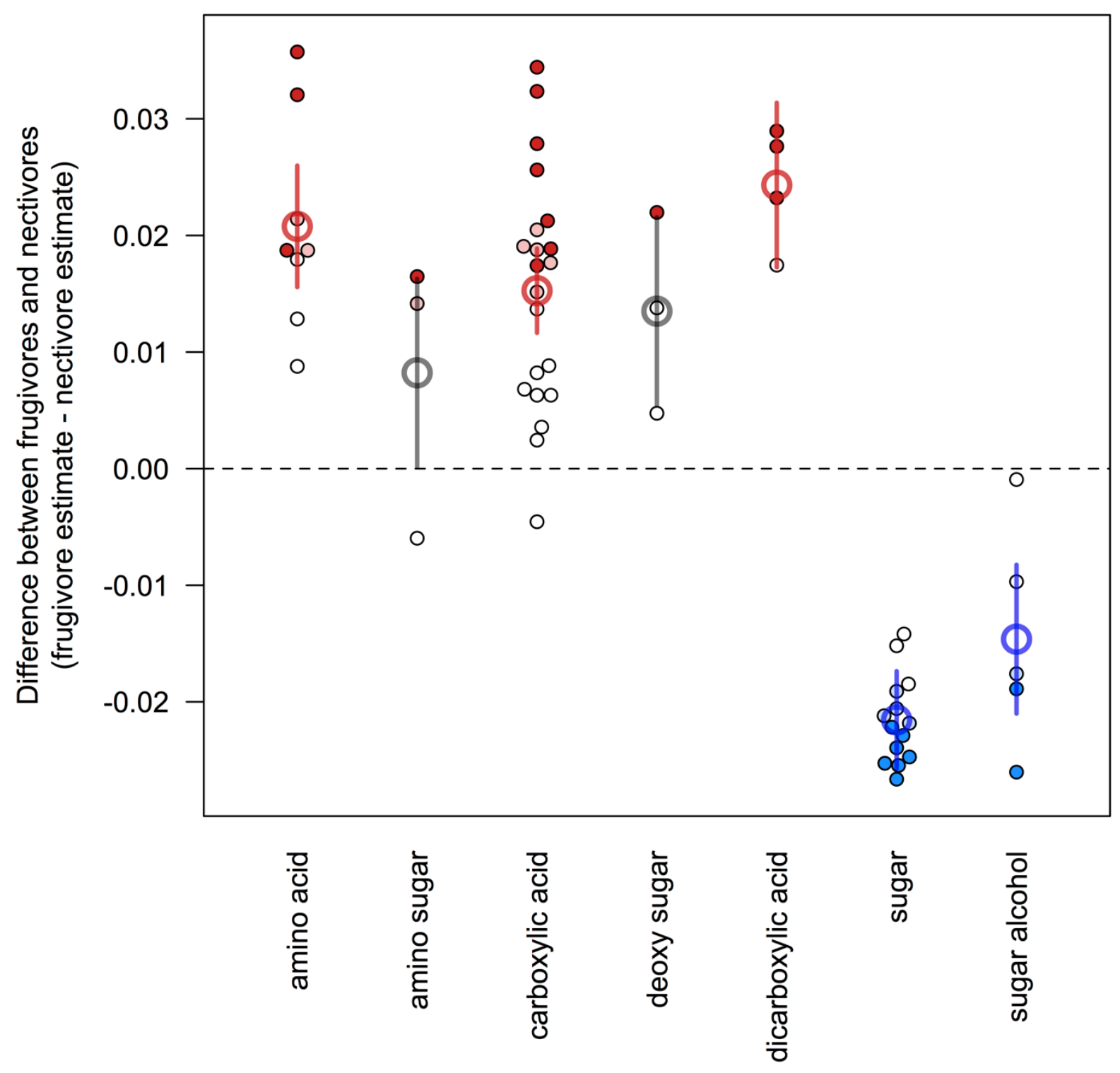

